\title{
Hybrid Computational-Experimental
}

\section{Data-Driven Design of Self-Assembling $\pi$-Conjugated Peptides}

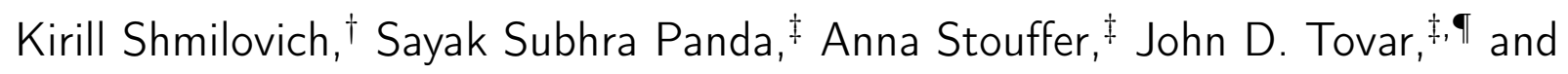
Andrew L. Ferguson ${ }^{*, \dagger}$

$\dagger$ Pritzker School of Molecular Engineering, University of Chicago, Chicago, Illinois 60637 $\ddagger$ Department of Chemistry, Johns Hopkins University, Baltimore, Maryland 21218

\Department of Materials Science and Engineering, Johns Hopkins University, Baltimore, Maryland 21218

E-mail: andrewferguson@uchicago.edu 


\begin{abstract}
Biocompatible molecules with electronic functionality provide a promising substrate for biocompatible electronic devices and electronic interfacing with biological systems. Synthetic oligopeptides composed of an aromatic $\pi$-core flanked by oligopeptide wings are a class of molecules that can self-assemble in aqueous environments into supramolecular nanoaggregates with emergent optical and electronic activity. We present an integrated computational-experimental pipeline employing all-atom molecular dynamics simulations and experimental UV-visible spectroscopy within an active learning workflow using deep representational learning and Bayesian optimization to design $\pi$-conjugated peptides programmed to self-assemble into elongated pseudo-1D nanoaggregtes with a high degree of H-type co-facial stacking of the $\pi$-cores. We consider as our design space the 694,982 unique $\pi$-conjugated peptides comprising a quaterthtiophene $\pi$-core flanked by symmetric oligopeptide wings up to five amino acids in length. After sampling only 1181 molecules $(\sim 0.17 \%$ of the design space) by computation and $28(\sim 0.004 \%)$ by experiment, we identify and experimentally validate a diversity of previously unknown high-performing molecules and extract interpretable design rules linking peptide sequence to emergent supramolecular structure and properties.
\end{abstract}




\section{Introduction}

Self-assembling $\pi$-conjugated peptides containing a $\pi$-core flanked by peptide wings represent a highly tailorable molecular building block for the bottom-up self-assembly of biocompatible supramolecular networks capable of long-range charge transport. ${ }^{1-12}$ Specific peptide sequences can promote secondary structures within the multi-molecular assemblies akin to beta sheets and guide the quadrupolar association of the $\pi$-cores into $\pi$-stacked nanostructures with long-range electronic delocalization. Wielding control over the molecular self-assembly of these nanomaterials to tailor the emergent structural, optical, and electronic properties can enable their functional applications as biocompatible, peptide-based field-effect transistors, photoconductors, or solar cells. ${ }^{2,4,7,13}$ The availability of 20 distinct natural amino acids and various $\pi$-cores make these systems extremely tunable and versatile in their optical and electronic properties. ${ }^{14-16}$ For example, we have previously tuned the steric volume of amino acids directly adjacent to the $\pi$-core to engineer tighter or looser packing of the assemblies $^{15,17}$ and controlled the supramolecular chirality of the nanoaggregates by modulating the length of an alkyl spacer between the peptide wings and the $\pi$-core. ${ }^{18}$ However, most of these materials have been developed by serendipity, intuition, or minor iterative modifications of existing molecules. Systematic data-driven screening approaches present the potential for much deeper and more efficient exploration of sequence space and the discovery of molecules with superior structural and functional properties.

The primary goal of the present work is to discover members of the $X_{n}$-quaterthtiophene$X_{n}\left(X_{n}-4 \mathrm{~T}-X_{n}\right)$ family of $\pi$-conjugated peptides capable of self-assembling into pseudo-1D nanoaggregates with in-register stacking of the quaterthtiophene $\pi$-cores guided by the $X_{n}$ peptide wings containing $n=1-5$ amino acids. Overlap of the $\pi$-cores is a structural prerequisite to supramolecular $\pi$ electron delocalization and the emergence of charge transport functionality. We chose to explore the quaterthiophene $\pi$-core due to its demonstrated applications in organic electronic field-effect transistors and photovoltaics ${ }^{19,20}$ and also our pre-

vious measurements of high charge mobilities in oligopeptide-quaterthiophene conjugates. ${ }^{14}$ 
The peptide wing containing $n=1-5$ amino acids is denoted as $X_{n}$, where each $X$ is one of the 20 natural amino acids. We limit the wing length to a maximum of five residues in order to simplify synthetic manipulation. We place two additional design constraints on the peptide wings. First, we require the oligopeptides to be head-to-tail invariant such that they are chemically symmetric about the quaterthtiophene core: one peptide wing is mirror symmetric to the other and each molecule possesses two C-termini. This symmetry imposes parity between the left and right sides of the molecular building blocks to promote the formation of linear supramolecular aggregates. Second, we require that the $X_{n}$ sequence contain at least one acidic residue (i.e., Asp, Glu) and no basic residues (i.e., Arg, His, Lys). The acidic side-chains together with the two carboxyl C-termini allows us to wield pH control of the oligopeptide protonation state such that they possess a formal negative charge of at least (-4)e at high $\mathrm{pH}$ and are formally charge neutral at low $\mathrm{pH}$. It has been previously shown through molecular modeling calculations and fluorescence correlation spectroscopy that intermolecular Coulombic repulsion and enhanced molecular solvation at high $\mathrm{pH}$ disfavors assembly and maintains the system as a mixture of monomers and small oligomers. ${ }^{21}$ Acidification protonates the ionizable groups to eliminate the Coulombic repulsion and serves as a trigger for large-scale supramolecular assembly. Under these two constraints, the $X_{n}-4 \mathrm{~T}-X_{n}$ family comprises 694,982 unique molecules possessing oligopeptide wings containing between $n=1-5$ amino acids. The design challenge is to discover the members of this design space that self-assemble into the most highly ordered linear nanoaggregates.

The large volume of sequence space means that no more than a tiny fraction of molecular candidates can be experimentally explored due to the time and labor costs associated with oligopeptide synthesis and assays. Edisonian trial-and-improvement experimental search is therefore highly inefficient and limited. Chemical intuition can help focus the search, but prior knowledge is restricted to a small number of previously studied candidates and also introduces human bias that can impede the discovery of high-performing, non-intuitive solutions. Computational modeling presents a means to conduct high throughput in silico screen- 
ing of molecular space. For example, Frederix et al. identified design rules for the assembly of tripeptide sequences by exhaustively simulating all possible amino acid combinations using coarse grained molecular dynamics simulation. ${ }^{22}$ For more complicated molecules and larger molecular search spaces, exhaustive enumeration becomes intractable and it is profitable to combine computational screening with data-driven modeling and active learning. The essence of this approach is to train on-the-fly sequence-property relationships over all computational screening data collected to date and use these models to guide subsequent rounds of the computational screen within a virtuous feedback loop. ${ }^{23-26}$ For example, Li et al. used machine learning algorithms such as random forests, gradient boosting, and logistic regression to predict the assembly and formation of hydrogels from possible peptidic precursors. ${ }^{27}$ Nagasawa et al. employed artificial neural networks and random forests for the discovery of conjugated polymers for organic photovoltaic applications. ${ }^{28}$ In the context of $\pi$-conjugated peptides, we previously combined coarse-grained molecular simulation with deep representational learning and Bayesian optimization to identify molecules predicted to exhibit superior assembly into pseudo-1D linear aggregates ${ }^{29}$ and we recently synthesized and tested perylene dimide based peptide- $\pi$ conjugated materials based on quantitative structure property relation models trained over molecular simulation data. ${ }^{17,30}$

A deficiency of data-driven virtual screening is the weak coupling between computation and experiment. High-throughput virtual screening using computation is used as an initial coarse filtration of the design space that identifies a manageably small number of candidates for synthesis and testing in a subsequent low-throughput experimental screen. ${ }^{31,32}$ The serial nature of this process means that there is no provision to incorporate experimental feedback into the data-driven search of the design space. This is a lost opportunity since the experimental data can serve as a source of high-quality information to better guide the search and correct for approximations and uncertainties inherent in the computational models. A hybrid data-driven search comprising parallel computational and experimental screens has the potential to offer the best of both worlds - high-throughput approximate computation 
to achieve broad coverage of the design space and low-throughput experimentation directed towards the most promising candidates. The enabling component of such a procedure is a data-driven model capable of constructing on-the-fly sequence-property relationships from experimental and computational screens that operate asynchronously and in parallel and measure/predict different properties of the molecular system. The trained model is then used within an active learning paradigm to select the most promising molecules for subsequent rounds of computational and experimental screening.

In this work, we develop and deploy a hybrid computational/experimental active learning approach for the data-driven design of $X_{n}-4 \mathrm{~T}-X_{n} \pi$-conjugated oligopeptides capable of self-assembling into pseudo-1D linear aggregates. We perform high-throughput computational screening using all-atom molecular dynamics simulations that predict the structural morphology of the self-assembled oligopeptide nanoaggregates. We conduct low-throughput experimental oligopeptide synthesis and characterize their assembly using UV-visible spectroscopy. We integrate the computational and experimental screening results to construct on-the-fly sequence-property models that performs asynchronous on-demand selection of the next batch of samples for computational or experimental screening. After sampling only $1181(\sim 0.17 \%)$ of the 694,982 molecules in the design space by computation and only 28 $(\sim 0.004 \%)$ by experiment, we discover and experimentally validate a diversity of previously

unknown high-performing oligopeptides capable of spontaneously assembling supramolecular aggregates with a high degree of H-type character and extract interpretable design rules linking peptide sequence to emergent supramolecular structure and properties.

\section{Methods}

In this section, we first present a high-level overview of the hybrid computational/experimental active learning workflow schematically illustrated in Fig. 1. We then present the methodological details of each component. For the interested reader, a more comprehensive explication 
of the theoretical underpinnings of these techniques, discussion of their numerical implementation, and the codes used to conduct this work are provided in the Supporting Information: Supporting Methods. ${ }^{33}$

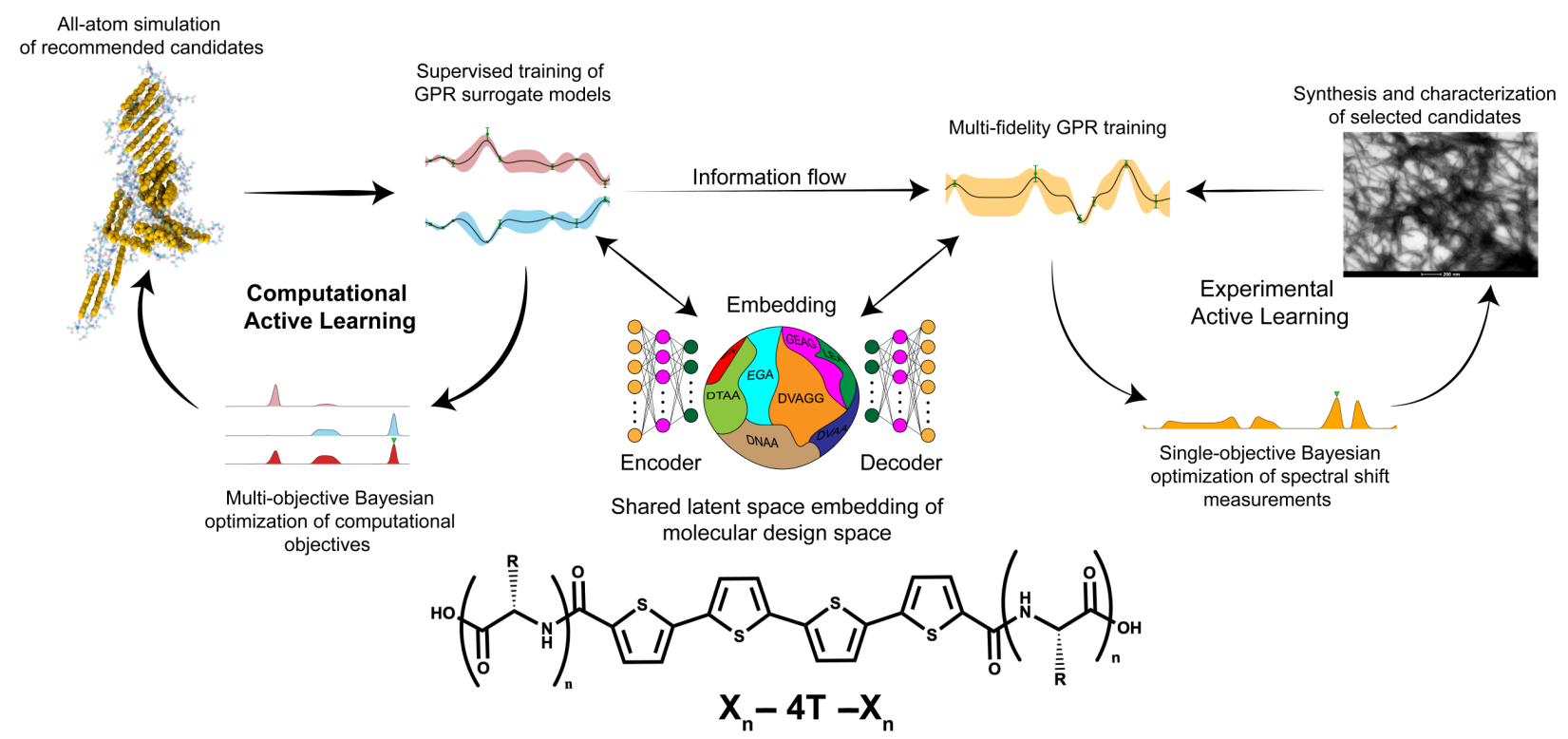

Figure 1: Schematic of the hybrid computational/experimental active learning workflow for the discovery of self-assembling $X_{n}-4 \mathrm{~T}-X_{n} \pi$-conjugated peptides. We perform separate computational and experimental active learning loops within a shared low-dimensional latent space embedding of the molecular design space learned using regularized autoencoders (RAE). Each active learning loop then consists of three parts. (i) Evaluating the quality of a given molecule $k$ in the $X_{n}-4 \mathrm{~T}-X_{n}$ design space by either performing highthroughput all-atom molecular dynamics simulations to measure the average number of contacts per molecule $\kappa^{(k)}$ and radius of gyration $R_{g}{ }^{(k)}$ of the self-assembled nanoaggregate or low-throughput experimental synthesis and measurement of the blue-shift $\lambda^{(k)}$ in the mode of the UV-vis spectrum. (ii) Fitting surrogate models using Gaussian process regression (GPR) to predict the performance of untested candidates given the accumulated simulation and experimental data collected to date. Two separate GPRs are maintained for the two computational objectives $\mathrm{GPR}_{\kappa}$ and $\mathrm{GPR}_{R_{g}}$. Importantly, we build a multi-fidelity GPR (mfGPR) as our experimental surrogate model $\mathrm{GPR}_{\lambda}$ that also incorporates data from the computational GPRs to improve prediction accuracy beyond what would be possible from the limited experimental data alone. (iii) Employing Bayesian optimization (BO) to interrogate the GPR model and select the next most promising molecular candidates for computation as those lying on the $\kappa-R_{g}$ Pareto frontier and for experimentation as those with large values of $\lambda$. The molecular renderings in this figure, and throughout the paper, are generated using the Visual Molecular Dynamics (VMD) software. ${ }^{34}$ 


\subsection{Overview}

We operate a high-throughput computational screening loop to perform all-atom molecular dynamics (MD) simulations to predict the structural morphology of the self-assembled nanoaggregates produced by particular $X_{n}-4 \mathrm{~T}-X_{n}$ sequences. The results of the computational screen are used to fit two surrogate sequence-property models relating oligopeptide sequence to the radius of gyration $R_{g}$ and number of intermolecular contacts $\kappa$ within the structure. The computational loop seeks to simultaneously maximize $\kappa$ and $R_{g}$ to produce pseudo-1D nanoaggregates with in-register $\pi$-stacking. We do not a priori know the appropriate relative weights of $\kappa$ and $R_{g}$ and so adopt an a posteriori optimization strategy in which we map out the family of Pareto optimal solutions populating the $\kappa-R_{g}$ Pareto frontier. ${ }^{35}$ We construct the sequence-property models over a low-dimensional embedding of the molecular design space extracted using regularized autoencoders ${ }^{36}$ (RAE) and fit the models using Gaussian process regression ${ }^{37}$ (GPR). The GPR predictions are passed to multi-dimensional Bayesian optimization ${ }^{38,39}$ (BO) routines to select the next most promising molecules to simulate for the computational screen.

We simultaneously operate a low-throughput experimental screening loop. In this loop, we synthesize $X_{n}-4$ T- $X_{n}$ oligopeptides and characterize their assembly by the blue-shift $\lambda$ in the mode of the UV-visible spectrogram between the unassembled (high-pH) and assembled (low-pH) states as a quantitative measure of the degree of H-type (i.e., co-facial) aggregation. Again we construct a surrogate sequence-property model relating oligopeptide sequence to the spectral shift where we use the same low-dimensional embedding furnished by the RAE. Importantly, the regression model we use to fit this relationship is a multi-fidelity GPR $(\mathrm{mfGPR})^{40}$ that we train over both the experimental measurements and the computational predictions. Despite measuring different observables of the molecular system $-\kappa$ and $R_{g}$ vs. $\lambda$ - the mfGPR can make use of the voluminous computational predictions to supplement the scarce experimental measurements to furnish a higher accuracy sequence-property model for the spectral shifts than would be possible using the experimental data alone. The com- 
putational and experimental screening loops operate asynchronously and in parallel and the data-driven GPR models are continually updated with each new batch of screening data.

The goal of the hybrid computational/experimental screening process is to discover and experimentally validate $X_{n}-4 \mathrm{~T}-X_{n}$ molecules with unprecedentedly large values of $\lambda$ indicative of exceptional in-register $\pi$-stacking and H-type character that is a prerequisite for supramolecular electronic delocalization and emergent optical and electronic functionality.

\subsection{All-atom molecular dynamics simulations}

All-atom molecular dynamics simulations of $X_{n}-4 \mathrm{~T}-X_{n}$ molecules were conducted using the GROMACS 2019.2 simulation suite. ${ }^{41}$ Simulations were initialized in the unassembled state by randomly placing $24 X_{n}-4 \mathrm{~T}-X_{n}$ molecules within a $10 \times 10 \times 10 \mathrm{~nm}^{3}$ simulation box with three-dimensional periodic boundary conditions and then solvating the system with TIP3P water. ${ }^{42}$ Peptides were modeled in the electrically neutral state corresponding to low-pH conditions and treated with the AMBER99SB-ILDN forcefield. ${ }^{43}$ The system was relaxed to $T=300 \mathrm{~K}$ and $P=1$ bar by steepest descent energy minimization, NVT equilibration ${ }^{44}$ and $\mathrm{NPT}^{45}$ equilibration. We subsequently conducted $200 \mathrm{~ns}$ NPT production runs to observe the spontaneous assembly of supramolecular nanoaggregates. Production runs were of sufficient duration that structural metrics of nanoaggregate formation stabilized over the course of the run. Simulation snapshots were extracted and saveed every 1 ps for analysis.

The fitness of a particular $X_{n}-4 \mathrm{~T}-X_{n}$ molecule was defined within our molecular simulations based on its ability to form pseudo-1D linear nanoaggregates with in-register stacking of the $\pi$-cores. We quantified this structurally via the the average number of contacts per molecule $\kappa$ and the radius of gyration $R_{g}{ }^{46}$ of the self-assembled nanoaggregates averaged over the terminal $50 \mathrm{~ns}$ of our production runs. An intermolecular contact is defined according to

our previously reported "optical distance" $d_{i, j}^{\text {optical }}$ that measures the minimum intermolecular distance between any pair of aromatic rings within the $\pi$-cores of molecules $i$ and $j .{ }^{29,47-49}$ We have previously shown that adopting a threshold of $d_{i, j}^{\text {optical }}<0.7 \mathrm{~nm}$ by which to define 
an intermolecular contact assures close proximity and in-register $\pi$-stacking between at least

one pair of aromatic rings. ${ }^{29,48-50}$ Maximizing $\kappa$ promotes $\pi$ - $\pi$ stacking while simultaneously maximizing $R_{g}$ encourages the formation of high-aspect ratio linear nanoaggregates. In general, we found maximization of either objective function alone was insufficient to promote in-register stacked pseudo-1D nanoaggregates: high $\kappa$ in the absence of high $R_{g}$ corresponds to globular structures with promiscuous multi-molecular $\pi$-stacking, whereas high $R_{g}$ in the absence of high $\kappa$ corresponds to weakly associated elongated threads lacking $\pi$-core overlaps. As we will show, the hybrid computational/experimental active learning framework learns the appropriate balance between $\kappa$ and $R_{g}$ that is most predictive of high-performing experimental candidates with large values of $\lambda$.

\subsection{Chemical space embedding}

Each $X_{n}-4 \mathrm{~T}-X_{n}$ molecule in our design space is differentiated by the identity of the peptide wing containing between one and five amino acids. We represent each candidate molecule as a linear amino acid graph where each node is an amino acid and the edges reflect their linear connectivity (Fig. S1 in the Supporting Information). The molecular design space of 694,982 molecules is large, discrete, and high-dimensional. It is possible to perform active learning directly over this space using, for example, kernel models, ${ }^{23,51-56}$ but superior search efficiencies can be achieved by first projecting the molecular design space into a smooth, continuous, low-dimensional space that is more amenable to the construction of robust regression models and deployment of optimization algorithms. ${ }^{26,29}$ We learn a bespoke latent space for the $X_{n}-4 \mathrm{~T}-X_{n}$ family using a regularized autoencoder ${ }^{36}$ (RAE), a deterministic variant of the popular variational autoencoder (VAE) architecture, ${ }^{57}$ trained on our entire molecular design space (Fig. S2). This encoder-decoder architecture consists of a message passing neural network ${ }^{58,59}$ encoder and a decoder performing explicit graph matching to ensure end-to-end invariance of node permutations. The encoder ${ }^{60-62}$ accepts graphical representations of the $X_{n}$ peptide sequences the nodes of which are featurized by the 553 single amino acid physio- 
chemical properties contained in the AAindex database and the edges of which are featurized by the 135 pairwise amino acid contact potentials and mutation matrices. ${ }^{63}$ The bottleneck layer defining the interface between the trained encoder and decoder contains a nonlinear latent embedding of the molecular design space $\boldsymbol{\xi}$ learned by the encoder that preserves relationships between molecular candidates and is sufficiently informative for the decoder to accurately reconstruct the molecular graphs (Fig. S3). We determine an appropriate dimensionality $d=32$ for the latent space using exploratory hyperparamter tuning optimizing the RAE reconstruction loss. ${ }^{64,65}$ This low-dimensional latent space defines a smooth and continuous representation $\boldsymbol{\xi}$ of the molecular design space over which fit (mf)GPR surrogate models and conduct BO-enabled active learning.

\subsection{Computational active learning}

The goal of the computational active learning loop is to drive computational discovery of candidate $X_{n}-4 \mathrm{~T}-X_{n}$ molecules that simultaneously maximize the average number of contacts per molecule $\kappa$ and the radius of gyration $R_{g}$ of the self-assembled aggregates. This amounts to searching for molecules residing upon the $\kappa-R_{g}$ Pareto frontier, for which the dual objective functions necessitate a multi-objective optimization strategy. We seeded the active learning screen by conducting MD simulations of 228 initial candidate molecules. To ensure broad initial sampling of the candidate space, we selected 100 molecules as those residing closest to the centroids of a 100-cluster k-means partitioning of the RAE latent space. The remaining 128 molecules were hand-selected to comprise a diversity of peptide wing lengths, residue hydrophobicity, aromaticity, polarity, and presence of heteroatoms. We used the computational predictions of $\kappa$ and $R_{g}$ to train two independent Gaussian process regression (GPR) surrogate models ${ }^{37} \hat{\kappa}=f(\boldsymbol{\xi})$ and $\hat{R}_{g}=g(\boldsymbol{\xi})$ that perform supervised learning of mappings from the latent space coordinates to the two structural observables. The predictions of the two GPRs are passed to a multi-objective BO routine that seeks to simultaneously maximize $\kappa$ and $R_{g}$ using the method of random scalarizations. ${ }^{38,39}$ The trained GPRs for 
$\kappa$ and $R_{g}$ are used to construct two independent BO upper confidence bound (UCB) ${ }^{66}$ acquisition functions defining the relative desirability of each candidate $X_{n}-4 \mathrm{~T}-X_{n}$ molecule in maximizing each of these two objectives. We then collapse these two acquisition functions into a single scalarized acquisition function constructed as a randomly weighted linear sum. The scalarized acquisition function is then used to perform univariate Bayesian optimization. A particular random scalarization corresponds to a particular choice of relative weightings between the two design objectives and defines a vector within the $2 \mathrm{D} \kappa-R_{g}$ space along which to maximize. Under sufficiently many repeated random scalarizations, the random vectors span the $\kappa-R_{g}$ Pareto frontier to discover a family of Pareto optimal solutions. We perform batched selection over different random scalarizations and choices of the UCB hyperparameter balancing the BO exploit-explore tradeoff to propose 25 new candidate molecules per round. MD simulations of these 25 molecules are performed and the three part active learning cycle is iteratively repeated. We assess convergence of the iterative screen by monitoring the set of Pareto optimal points that define the $\kappa-R_{g}$ Pareto frontier and terminate sampling once the Pareto frontier ceases to advance with additional rounds of sampling. We conducted 38 rounds of computational screening over which we considered 1181 candidate molecules. A full accounting of the molecules identified in each round of the computational active learning loop is provided in the Supporting Information: Data Availability. ${ }^{33}$

\subsection{Experimental active learning}

The experimental active learning loop aims to maximize the blue-shift $\lambda$ in the mode of the UV-visible spectrogram between the unassembled (high-pH) and assembled (low-pH) states. The magnitude of this spectral shift $\lambda$ has been experimentally shown to correlate with cofacial H-type assembly that results in formation of the desired pseudo-1D linear stacks. ${ }^{14,67,68}$ We seeded the experimental search with a set of 11 molecules hand selected from the 228 molecules comprising the initial computational round to comprise a diversity of oligopeptide wing lengths and predicted values of $\kappa$ and $R_{g}$. We trained a GPR model $\hat{\lambda}=h(\boldsymbol{\xi})$ to predict 
the spectral shift $\lambda$ as a function of latent space coordinates. In this case we have only a single objective function $\lambda$ but we wish to construct a multi-fidelity surrogate model incorporating both direct experimental measurements of $\lambda$ and computational predictions of $\kappa$ and $R_{g}$. The rationale is that the computational predictions for $\kappa$ and $R_{g}$ should be correlated with and predictive of the experimental measurements of $\lambda$. This is expected to be the case since $\kappa$ and $R_{g}$ are structural measures of the degree of in-register $\pi$-stacking in elongated nanoaggregates that are prerequisites for H-aggregate character manifested in measurements of $\lambda$. A multifidelity model trained to learn a nonlinear mapping from the low-fidelity computational predictions to high-fidelity experimental measurements can take advantage of the abundant computational data to produce a superior model than that obtained by training over only the sparse experimental data alone. Indeed, by the terminal round of experimental active learning, incorporation of computational screening data within the multi-fidelity paradigm leads to a $\sim 27 \%$ improvement in the predictive accuracy of our surrogate model compared to a single-fidelity model fitted only over the experimental observations (Fig. S4).

We construct multi-fidelity surrogate models fusing the computational (low-fidelity) and experimental (high-fidelity) data using the multi-fidelity Gaussian process regression (mfGPR) formalism. ${ }^{40}$ The mfGPR model is then passed to a standard BO routine ${ }^{38}$ employing an expected improvement (EI) acquisition function ${ }^{38,69,70}$ and the Kringing believer ${ }^{71}$ batched $^{2}$ sampling. We use the BO to propose a batch of molecules for the next round of sampling, which we manually down-select to 8-9 molecules for experimental synthesis and characterization. By incorporating "human-in-the-loop" curation of the selected molecules we hope to balance purely data-driven candidate proposal with chemical intuition and thereby incorporate some degree of prior knowledge and human experience into the search process without, we hope, corrupting the search with too much bias. The success of this collaborative human-machine paradigm has been previously demonstrated in the data-driven discovery of molecular organic light emitting diodes. ${ }^{32}$ The experimental measurements are fed back into the low-throughput experimental active learning loop that is executed asynchronously and 
in parallel with the high-throughput computational loop. We execute three rounds of experimental active learning over the course of the 38 rounds of computational active learning that are executed at computational rounds 0, 14, and 22. Given the good performance of the candidates studied in the third experimental round together with the relatively modest advances in the Pareto frontier observed over computational rounds 23-38, we elected to terminate our experimental screen after its third round. A full accounting of the molecules identified in each round of the experimental active learning loop is provided in Table 1 and in the Supporting Information: Data Availability. ${ }^{33}$

\subsection{Nonlinear manifold learning of low-dimensional assembly path- ways}

After completing the hybrid computational/experimental screen we subjected the ensemble of 1181 molecular simulation trajectories of $X_{n}-4 \mathrm{~T}-X_{n}$ candidate molecules to nonlinear dimensionality reduction in order to resolve the structural assembly pathways. In doing so, we sought to gain mechanistic understanding of the molecular assembly mechanisms differentiating the top performing molecules identified by our screen. We performed nonlinear dimensionality reduction using diffusion maps manifold learning ${ }^{72,73}$ to project the configurational coordinate space into a low-dimensional space preserving the leading high-variance collective

dynamics of the system. ${ }^{29,47,74-77}$ Diffusion maps take as an input a pairwise distance matrix measuring the configurational similarity between all 118,100 simulation snapshots harvested from the ensemble of 1181 simulation trajectories. We define these pairwise distances using the smooth overlap of atomic positions (SOAP) kernel ${ }^{78-81}$ between the heavy atoms constituting the $4 \mathrm{~T} \pi$-cores as a distance metric that is naturally invariant to rotations, translations, and permutations of atoms, and which - as a $\pi$-core-centric metric - can be applied between oligopeptides with different wing lengths. The influence of the wings is implicitly retained through their impact on the configurations adopted by the $\pi$-cores. A density-adaptive variant of diffusion maps ${ }^{82}$ is then applied to furnish embeddings of the as- 
sembly trajectories into a 2D manifold that exposes the assembly pathways and mechanisms followed by the various $X_{n}-4 \mathrm{~T}-X_{n}$ molecules.

\subsection{Oligopeptide synthesis}

General Information. $N, N$-Dimethylformamide (DMF) was purchased from Sigma-Aldrich. N-Methyl-2-pyrrolidone (NMP) was obtained from Advanced ChemTech. Dichloromethane (DCM) and $n$-hexane were freshly distilled prior to storage. All solvents were stored over 4 $\AA$ molecular sieves and were subsequently degassed by sparging with nitrogen gas at least 30 min prior to use. O-(Benzotriazol-1-yl)- $N, N, N^{\prime}, N^{\prime}$-tetramethyluronium hexafluorophosphate (HBTU) was purchased from Oakwood Products Inc. Tetrakis(triphenylphosphine)palladium was obtained from Strem Chemicals. Wang resin (preloaded with amino acid) and Fmocprotected amino acids were obtained from Advanced Chem Tech. 5-Bromo-2-thiophenecarboxylic acid was obtained from Accela Chem Bio Co. Ltd. All other reagents and starting materials were obtained from Sigma-Aldrich and were used as received. Details of the synthesis for individual oligopeptides is provided in the Supporting Information: Peptide Synthesis.

Electrospray Ionization Mass Spectrometry (ESI-MS). ESI samples were collected using a Thermo Finnigan LCQ Deca Ion Trap Mass Spectrometer in negative mode. Samples were prepared in a 1:1 MeOH:water solution with 1\% ammonium hydroxide. ESI spectra for each synthesized peptide are provided in the Supporting Information: ESI Spectra.

Reverse-Phase High Performance Liquid Chromatography (HPLC). HPLC purification was performed on an Agilent 1100 series (semipreparative/analytical) and a Varian PrepStar SD-1 (preparative) instrument using Luna $5 \mu \mathrm{m}$ particle diameter C8 with TMS end-capping columns with silica solid support. An ammonium formate aqueous buffer (pH 8) and acetonitrile were used as the mobile phase. HPLC traces for each synthesized peptide are provided in the Supporting Information: Analytical HPLC Traces.

General Solid-Phase Peptide Synthesis (SPPS). All peptides were synthesized us- 
ing the standard Fmoc solid-phase technique with Wang resin preloaded with Fmoc-protected amino acids. To the resin in a peptide chamber, Fmoc-deprotection was accomplished by adding a $20 \%$ piperidine solution in DMF twice (successive 5- and 10-min treatments) followed by washing with $\mathrm{NMP} \times 3$, methanol $\times 3$, and $\mathrm{DCM} \times 3$. For the amino acid couplings, 3.0 equiv of the Fmoc-protected amino acid was activated with 2.9 equiv of HBTU and 10 equiv of diisopropylethylamine (DIPEA) in NMP, and this solution was added to the resin beads. The reaction mixture was allowed to mix for 45-60 min, after which the beads were rinsed with NMP, methanol, and DCM (3 times each). The completion of all couplings was monitored using a Kaiser test on a few dry resin beads, repeating the same amino acid coupling if needed. The general procedure for amino acid coupling was repeated for each additional amino acid until the desired peptide sequence was obtained.

General $N$-Acylation Procedure for Peptides. Following our previous procedure, ${ }^{18}$ a solution containing 3 equiv of 5-Bromo-2- thiophenecarboxylic acid, HBTU (2.9 equiv), and DIPEA (10 equiv) in NMP was mixed with the oligopeptide-bound resin for $3 \mathrm{~h}$. The completion of the $N$-acylation was assessed using a Kaiser test on a few dry resin beads. The resin was washed with NMP, methanol, and DCM (3 times each).

General On-Resin Stille Coupling Procedure. The solid-supported $N$-acylated oligopeptide (1 equiv) was transferred to a Schlenk flask equipped with a reflux condenser. The resin was dried under vacuum. $\mathrm{Pd}\left(\mathrm{PPh}_{3}\right)_{4}(4 \mathrm{~mol} \%$, relative to resin loading) was added to the reaction vessel. An approximately $15 \mathrm{mM}$ solution of the 5,5'-bis-trimethylstannyl$\left[2,2^{\prime}\right]$-bithiophene (0.50 equiv) was prepared in DMF and was added to the reaction flask via syringe. The mixture was heated to $80^{\circ} \mathrm{C}$ for $18 \mathrm{~h}$ and was agitated constantly by bubbling nitrogen through the solution. The mixture was allowed to cool to room temperature. The peptide was subjected to the general cleavage and workup procedure to yield the crude product and then further purified by HPLC.

General Peptide Cleavage and Workup Procedure. Following dimerization, the resin was returned to the peptide chamber and again subjected to a wash cycle: $2 \times \mathrm{NMP}$, 
$2 \times$ methanol, and $2 \times \mathrm{DCM}$. The resin was then treated with $9.5 \mathrm{~mL}$ of trifluoroacetic acid, $250 \mu \mathrm{L}$ of water, and $250 \mu \mathrm{L}$ of triisopropylsilane for $3 \mathrm{~h}$. The peptide solution was filtered from the resin beads, washed three times with DCM, and concentrated by evaporation under reduced pressure. The crude peptide was then precipitated from the solution with 40-50 mL of diethyl ether and isolated through centrifugation. The resulting pellet was triturated with diethyl ether to yield the crude product, which was dissolved in approximately 20-25 mL of water. $30 \mu \mathrm{L}$ of potassium hydroxide $(1 \mathrm{M})$ was added if needed to solubilize the peptides in water and lyophilized.

\section{$2.8 \quad$ UV-visible spectroscopy}

UV-vis spectra were obtained using a Varian Cary 50 Bio UV-vis spectrophotometer. Spectroscopic samples were prepared by diluting the peptide solution to the appropriate concentration in Millipore water to achieve an optical density near 0.1, 0.2 , and 0.3 in the monomeric/basic solution. The $\mathrm{pH}$ was then adjusted by adding $20 \mu \mathrm{L}$ of $1 \mathrm{M} \mathrm{KOH}$ (basic) followed by addition of $40 \mu \mathrm{L}$ of $1 \mathrm{M} \mathrm{HCl}$ (acidic). Approximate concentration of the peptides were $2.25 \mu \mathrm{M}, 4.50 \mu \mathrm{M}$, and $6.75 \mu \mathrm{M}$ for optical density of $0.1,0.2$, and 0.3 respectively.

\section{$3 \quad$ Results and Discussion}

\subsection{Hybrid computational/experimental active learning discovers novel high-performing oligopeptides}

We report in Fig. 2 the results of our hybrid computational/experiment active learning screen to the molecular design space of $694,982 X_{n}-4 \mathrm{~T}-X_{n}$ candidate molecules. We conduct 38 rounds of computational screening to simulate a total of $1181 X_{n}-4 \mathrm{~T}-X_{n}$ molecules inter-

leaved with three rounds of experimental screening in which we synthesize and test a total of 28 molecules. A full accounting of the molecules identified in each round of the compu- 
tational and experimental active learning loops are provided in the Supporting Information: Data Availability. ${ }^{33}$ Round 0 of the computational and experimental screens commence simultaneously, respectively screening 228 and 11 molecules. The two screens then proceed asynchronously and in parallel. The high-throughput computational loop considers 25 candidate molecules per round and iterates more rapidly than the low-throughput experimental screen that considers 8-9 molecules per round. We track the progress of the experimental screen via the measured spectral blue shifts $\lambda$ upon assembly as a measure of the prevalence of H-type co-facial $\pi$-stacking (Fig. 2a). We track the progress of the computational screen via the advancement in the $\kappa-R_{g}$ Pareto frontier that we quantify through the mean distance from the origin of all $n_{i}$ molecules cumulatively simulated over the first $i$ rounds (Fig. 2a,b),

$$
d_{\text {Pareto }}^{(i)}=\frac{1}{n_{i}} \sum_{k=1}^{n_{i}} \sqrt{\left(\kappa^{(k)}\right)^{2}+\left(R_{g}^{(k)}\right)^{2}}
$$

We recall that large values of the average number of $\pi$-core contacts per molecule $\kappa$ and radius of gyration of the self-assembled aggregates $R_{g}$ are anticipated to lead to correlate with the formation of pseudo-1D nanoaggregates with high degrees of H-type character.

Round 1 of the experimental screen is performed upon completing computational Round 14, at which time a total of 578 candidate molecules have been computationally assessed. These computational screening data are passed to the experimental surrogate model and Bayesian optimization within the multi-fidelity hybrid computational/experimental active learning framework in order to better inform the design of experimental Round 1 than would be possible by analyzing the 11 experimental data points alone. Under our human-in-theloop selection protocol, we selected nine molecules for Round 1 of experimental screening by filtering a 35-molecule list outputted from our BO routine. Down-selection was performed on the basis of high anticipated performance based on our previous experimental and computational work ${ }^{15,29}$ and maintenance of a diversity of peptide wing compositions and lengths. This human-in-the-loop selection process serves as a simple means to inject prior knowledge 


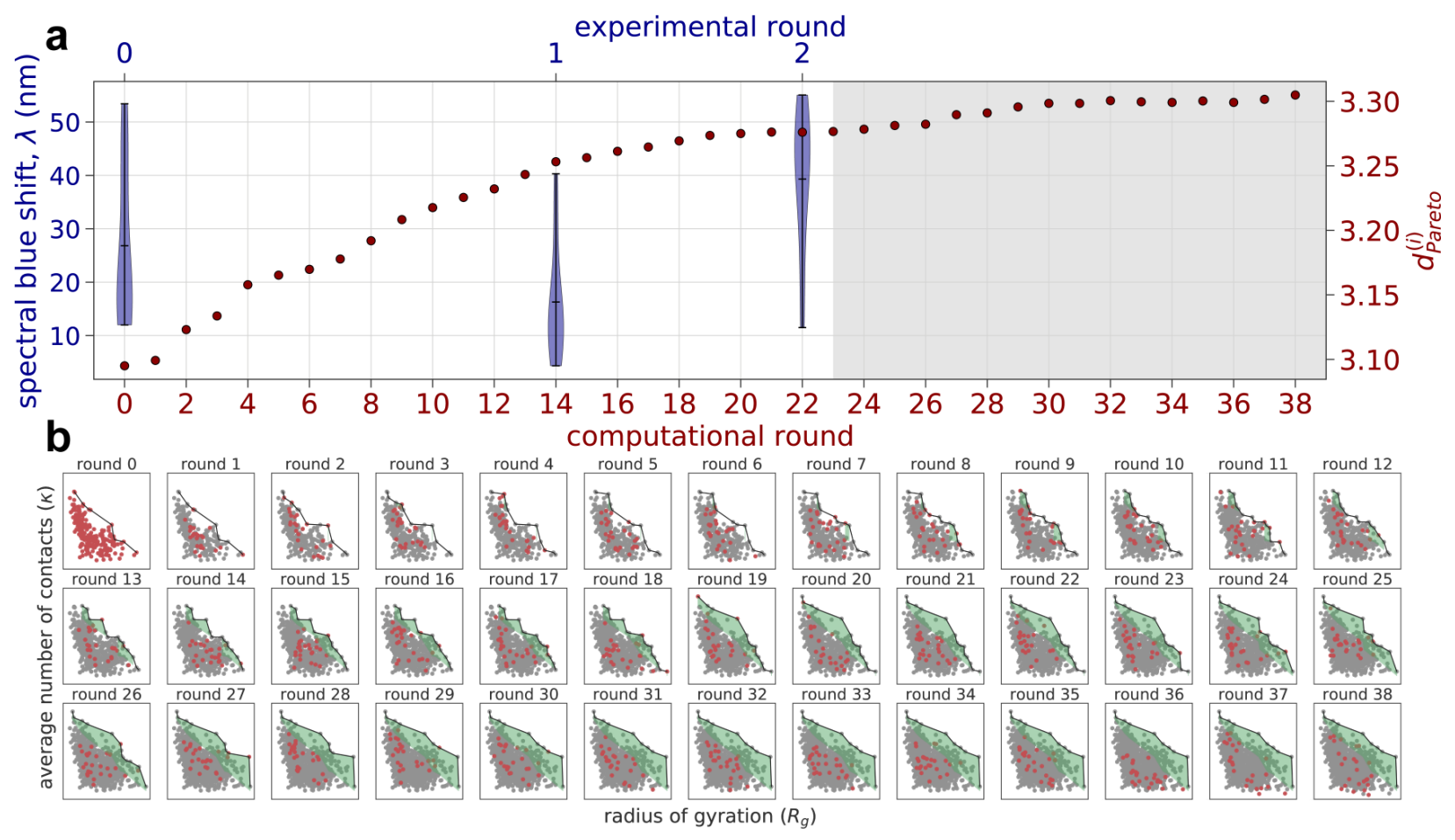

Figure 2: Progress and convergence of the hybrid computational/experimental active learning screen for high-performing $X_{n}-4 \mathrm{~T}-X_{n}$ molecules within the design space of 694,982 candidates. (a) A total of 38 computational rounds of screening are performed and interleaved with three rounds of experimental screening. The experimental screens are conducted at computational Rounds, 0,14 , and 22. Convergence of the computational screen is tracked by $d_{\text {Pareto }}^{(i)}$, the mean distance from the origin of all molecules cumulatively simulated over the first $i$ rounds, as a measure of the advancement of the optimal frontier (red circles). Convergence of the experimental screen is tracked by the measured spectral blue shift $\lambda$ quantifying the degree of $\mathrm{H}$-type co-facial $\pi$-stacking within the self-assembled nanoaggregates (blue violin plots). The grey shaded area represents the computational rounds used to verify convergence of the active learning but not used to inform any additional rounds of experimental design. (b) Round-by-round advancement of the computational $\kappa-R_{g}$ Pareto frontier over the course of the 38 screening rounds. Within each frame, the points corresponding to $X_{n^{-}}$ $4 \mathrm{~T}-X_{n}$ candidates collected within that round are shown in red, those points collected in previous rounds are shown in grey, the Pareto frontier defined by the Pareto optimal points is shown as a black solid line, and the shaded green area indicates the advancement of the Pareto frontier relative to Round 0.

into the data-driven search process, which can be particularly valuable in the early stages of the search where the models are trained over small numbers of data points, by directing the search process to regions of molecular design space that are anticipated to be particularly profitable. 
Round 2 of the experimental screen is conducted after completing computational Round 22, at which point we have simulated 780 candidate molecules. Again, the totality of these computational screening data are used to augment the multi-fidelity experimental surrogate model and used to pick eight candidate molecules for experimental testing down-selected from the 75 top candidates proposed by the BO routine. We inject one addition piece of human intuition into the down-selection process by making a single Tyr to Ala amino acid mutation of one of the predicted sequences - YEVGA to AEVGA - based on prior understanding that aromatic side chains are known to $\pi$-stack with the $\pi$-cores and therefore liable to disrupt favorable in-register $4 \mathrm{~T}$ stacking. ${ }^{29}$ This modification is substantiated by both observing low-ranking candidates possessing bulky aromatic residues in the first two experimental rounds (EYIQG: rank 18/28, VEF: rank 23/28, GFGFD: rank 25/28) along with previous experimental work noting the presence of aromatic residues resulting in reduced UV-vis blue-shifts. ${ }^{15}$

We continue to conduct an additional 16 rounds of computational screening (Rounds 23-38) while experimental Round 2 is being completed in anticipation of possibly conducting a fourth experimental round. After a relatively rapid growth in $d_{\text {Pareto }}$ over the course of computational Rounds $0-20$, we observed the emergence of a plateau at $d_{\text {Pareto }} \approx 3.3$ by Round 29 and only a $0.7 \%$ increase in $d_{\text {Pareto }}$ relative to Round 22 (Fig. 2a). This observation is mirrored by a relatively modest advancement of the Pareto frontier between Rounds 23-38 (Fig. 2b). Experimentally, the mean spectral shift $\lambda$ in experimental Round 2 is $46 \%$ better than Round 0, and the top performing Round 2 candidate is $3 \%$ superior to that in Round 0 . The diminishing returns evinced by the computational $d_{\text {Pareto }}$ and the successful discovery of a molecule with superior $\lambda$ impelled us to terminate our search after experimental Round 2 / computational Round 38.

In all, we simulated 1181 molecules comprising $\sim 0.17 \%$ of the 694,982 molecules constituting the $X_{n}-4 \mathrm{~T}-X_{n}$ design space, corresponding to $236.2 \mu$ s of simulation time, and requiring 4.97 GPU-years of parallel compute. Experimentally, we synthesized and char- 
acterized a total of $28 X_{n}-4 \mathrm{~T}-X_{n}$ molecules over the course of the course of eight months corresponding to exploration of $0.004 \%$ of the molecular space.

We present in Fig. 3 an embedding of all 1181 simulated molecules and 28 experimentally tested molecules into the $\kappa$ - $R_{g}$ objective function space used to identify high-performing molecules in the computational screening loop. Molecular renderings of the self-assembled nanoaggregates provides qualitative visual conformation that $X_{n}-4 \mathrm{~T}-X_{n}$ molecules producing aggregates with large values of both $\kappa$ and $R_{g}$ do indeed tend to self-assemble into pseudo-1D structures with good stacking of the $4 \mathrm{~T} \pi$-cores.

The primary result of our hybrid computational/experimental active learning screen are experimental measurements of $28 X_{n}-4 \mathrm{~T}-X_{n}$ molecules reported in Table 1 . Of these molecules, 26 are completely novel and on par with known high-performing sequences while also possessing greater diversity amino acid sequences previously unknown to correlate with good spectral blue shifts $\lambda$. The high values of $\lambda$ for these molecules are indicative of a high degree of H-type co-facial stacking and the potential for long-range supramolecular electronic delocalization and emergent optoelectronic functionality. Additionally, we were encouraged that our active learning procedure spontaneously discovered DGG-4T-GGD as a previously known high-performing candidate. ${ }^{68,83}$ A complete list of predicted $\kappa$ and $R_{g}$ values from the terminal computational surrogate model and predicted spectral shift measurements $\lambda$ from the terminal experimental surrogate model for all 694,982 molecules within the $X_{n}-4 \mathrm{~T}-X_{n}$ design space is also provided in the Supporting Information: Data Availability. ${ }^{33}$

\subsection{Molecular design rules}

Our rank-ordered list of 28 experimentally assayed candidates exposes a number of oligopeptide design precepts, that is relationships between the placement/omission of particular amino acids at specific positions along the oligopeptide wing and the magnitude of the spectral blue shift $\lambda$ quantifying the degree of H-type co-facial association within the selfassembled nanoaggregates. Despite the relatively small size of the experimental data set, we 


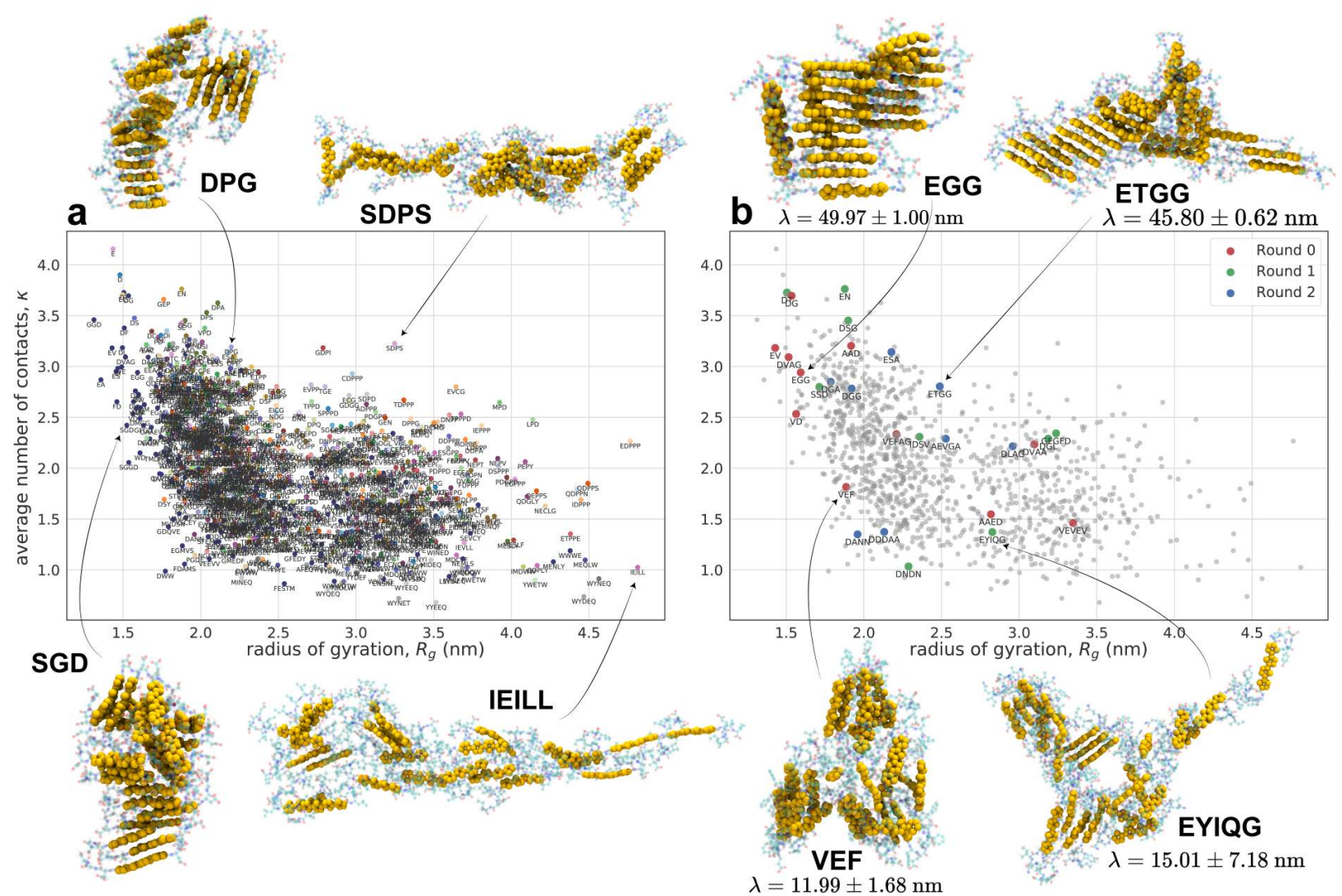

Figure 3: Embedding of the computational and experimental molecules sampled in the active learning screen into the $\kappa-R_{g}$ objective function space. (a) Embedding of the $1181 X_{n}-4 \mathrm{~T}-X_{n}$ molecules explored in the computational screen. Points shown in the same color were sampled during the same computational active learning round. The labels associated with each point corresponds to the $X_{n}$ peptide wing sequence. (b) Highlighting the 28 experimentally tested $X_{n}-4 \mathrm{~T}-X_{n}$ molecules (colored points) superposed onto all 1181 computationally simulated points (grey points). The color indicates the round of experimental active learning within which the molecule was tested. Encircling the plot are snapshots from our molecular simulation trajectories showing the terminal self-assembled nanoaggregates. The heavy atoms constituting $4 \mathrm{~T} \pi$-cores are rendered as gold space-filling spheres and the $X_{n}$ peptide wings as semi-transparent ball-and-stick representations. For the experimentally tested candidates we also report the measured values of the spectral blue shift $\lambda$. A full accounting of the computed $\kappa$ and $R_{g}$ values and measured $\lambda$ values for all molecules considered in our screen is provided in the Supporting Information: Data Availability. ${ }^{33}$

were able to extract three statistically significant design rules. First, the nine top-ranked molecules within the 28 assayed candidates contain a distal Asp or Glu residue at the C-terminus and a Gly or Ala residue in the position most proximate to the $\pi$-core. A 
Table 1: Rank-ordered list of the 28 experimentally tested $X_{n}-4 \mathrm{~T}-X_{n}$ molecules sampled over the course of the active learning screen.

\begin{tabular}{ccccc}
\hline Rank & Peptide wing, $X_{n}$ & Measured spectral shift, $\lambda(\mathrm{nm})$ & Discovery round & Previously known? \\
\hline 1 & DGG & $55.06 \pm 1.00$ & 2 & Yes $^{68,83}$ \\
2 & DG & $53.42 \pm 4.16$ & 0 & $\mathrm{~N}$ \\
3 & ESA & $50.01 \pm 0.53$ & 0 & $\mathrm{~N}$ \\
4 & EGG & $49.97 \pm 1.00$ & 2 & Yes $^{14}$ \\
5 & ETGG & $45.80 \pm 0.62$ & 2 & $\mathrm{~N}$ \\
\hline 6 & DGA & $44.15 \pm 1.49$ & 2 & $\mathrm{~N}$ \\
7 & DDDAA & $42.07 \pm 0.46$ & 0 & $\mathrm{~N}$ \\
8 & DVAA & $41.65 \pm 1.15$ & 1 & $\mathrm{~N}$ \\
9 & DSG & $40.32 \pm 1.15$ & 2 & $\mathrm{~N}$ \\
10 & AEVGA & $40.15 \pm 1.12$ & 0 & $\mathrm{~N}$ \\
\hline 11 & DVAG & $35.70 \pm 1.49$ & 1 & $\mathrm{~N}$ \\
12 & DNDN & $29.58 \pm 4.76$ & 2 & $\mathrm{~N}$ \\
13 & DANN & $25.70 \pm 0.32$ & 0 & $\mathrm{~N}$ \\
14 & VEFAG & $21.75 \pm 2.07$ & 0 & $\mathrm{~N}$ \\
15 & VEVEV & $18.43 \pm 0.62$ & 0 & $\mathrm{~N}$ \\
\hline 16 & VD & $18.02 \pm 2.66$ & 0 & $\mathrm{~N}$ \\
17 & AAD & $15.98 \pm 1.00$ & 1 & $\mathrm{~N}$ \\
18 & EYIQG & $15.01 \pm 7.18$ & 0 & $\mathrm{~N}$ \\
19 & EV & $14.70 \pm 1.20$ & 1 & $\mathrm{~N}$ \\
20 & DT & $14.70 \pm 1.20$ & 0 & $\mathrm{~N}$ \\
\hline 21 & AAED & $13.68 \pm 1.46$ & 1 & $\mathrm{~N}$ \\
22 & SSD & $13.68 \pm 1.15$ & 0 & $\mathrm{~N}$ \\
23 & VEF & $11.99 \pm 1.68$ & 2 & $\mathrm{~N}$ \\
24 & DLAG & $11.49 \pm 0.46$ & 1 & $\mathrm{~N}$ \\
25 & GFGFD & $10.97 \pm 1.77$ & 1 & $\mathrm{~N}$ \\
\hline 26 & DGL & $10.25 \pm 1.20$ & 1 & $\mathrm{~N}$ \\
28 & IDSV & $7.70 \pm 3.83$ & 0 & 2 \\
\hline
\end{tabular}

statistical analysis using a one-sided Mann-Whitney $\mathrm{U}_{\text {test }}{ }^{84}$ reveals a statistically significant $(\mathrm{p}=0.0001)$ increase in the measured blue shifts $\lambda$ associated with the presence of the $(\mathrm{D} / \mathrm{E}) X_{n}(\mathrm{~A} / \mathrm{G})$ motif. In our prior work on $\pi$-conjugated oligopeptides, we typically synthesized the peptide wings with the ionizable residue responsible for actuating $\mathrm{pH}$-triggered assembly located at the C-terminus to locate it as far away as possible from the $\pi$-core. Our motivation for this design choice was that the hydrophilic and polar nature of these residues which, together with their steric bulk, was anticipated to disrupt good supramolecular assembly of the $\pi$-cores. ${ }^{14,15,68,85-88}$ Interestingly, our active learning screen appears to 
have also learned this design rule without any explicit human instruction and furnished post hoc support for this intuitive choice. Similarly, our recent computational and experimental work. ${ }^{29,30,89}$ is consistent with prior chemical intuition ${ }^{85}$ that the placement of small nonpolar residues adjacent to the $\pi$-core should promote good co-facial stacking of the cores. Again, the active learning screen appears to have also learned this design rule within the sequence-property surrogate model and is consistent with a physical rationale that the small steric volume of these amino acids is conducive to in-register $\pi$-stacking of the $4 \mathrm{~T}$ cores. It is more challenging, however, to differentiate between the performance of Gly vs. Ala, with Gly leading to more favorable spectral shifts within a DGX motif $-\lambda_{\mathrm{DGG}}=(55.05 \pm 1.00)$ $\mathrm{nm}$ and $\lambda_{\mathrm{DGA}}=(44.15 \pm 1.49) \mathrm{nm}$ - whereas Ala performs better within a DVAX motif $\lambda_{\text {DVAG }}=(35.70 \pm 1.49) \mathrm{nm}$ and $\lambda_{\text {DVAA }}=(41.65 \pm 1.15) \mathrm{nm}$. The absence of a simple modular decomposition of the influence of each amino acid position in the $X_{n}$ wing reflects the complexity of the self-assembly process and the important role of multi-body interactions, amino acid context, and wing length.

Second, consistent with the favorability of core-adjacent Gly and Ala residues, the non-Cterminal amino acids within the $X_{n}$ peptide sequences of the top-performing molecules tend to also be enriched in small hydrophobic residues such as Ala, Gly or Val (one-sided MannWhitney $\mathrm{U}$ test, $\mathrm{p}$-value=0.004). Interestingly, residues containing polar hydroxyl groups such as Ser and Thr are also over-represented within high-performing sequences when Ser or Thr are non-terminal residues and Asp or Glu are C-terminal such as ESA: rank 3/28, ETGG: rank 5/28, and DSG: rank 9/28 (one-sided Mann-Whitney U test, p-value=0.03). Other polar residues like Asn also perform relatively well in the $\pi$-core proximate position when Asp occupies the distal slot (DNDN: rank 12/28; DANN: rank 13/28).

Third, the presence of larger hydrophobic and bulky aromatic residues such as Leu, Ile, Phe, and Tyr at any location are correlated with poorer performing candidates such as DLAG: rank 24/28, DGL: rank 26/28, EYIQG: rank 18/28, and IDSV: rank 27/28, with the poorest-performing candidates enriched in these four amino acids (one-sided Mann-Whitney 
$\mathrm{U}$ test, $\mathrm{p}$-value $=0.002$ ). We have previously shown that favorable interactions between these large hydrophobic residues and the aromatic $\pi$-cores can disrupt supramolecular association between the cores. ${ }^{29}$ This observation is also consistent with our prior observation that oligopeptides possessing oligophenylenevinylene $\pi$-cores exhibited larger spectral blue shifts when the peptide wings contained small Gly and Ala residues compared to larger Ile and Val residues. ${ }^{15}$

Finally, although no Pro containing molecules were sampled in the experimental screen, we note that a number of simulated molecules containing a Pro residue lie at or near the $\kappa-R_{g}$ Pareto frontier and were highly ranked in predicted blue shift $\lambda$ by the terminal mfGPR surrogate model (e.g., DPG: rank 329/694,982, AEPP: rank 183/694,982, SDPD: rank 10/694,982, EAP: rank 13/694,982, DDPA: rank 23/694,982, GEPG: rank 15/694,982). This finding is somewhat surprising because Pro has been largely unexplored in previous experimental and computational $\pi$-conjugated peptide studies. Proline, with its unique conformational properties including its conformational rigidity and absence of hydrogen bond donor capacity, appears to be quite favorable in promoting good in-register stacking between the $\pi$-cores and the formation of high-aspect ratio nanoaggregates. We suggest that experimental testing and further computational exploration of the molecular mechanisms underpinning these predictions may be a profitable avenue for future investigations.

\subsection{Molecular assembly pathways}

Having extracted design rules linking amino acid sequence to the degree of H-type stacking within the nanoaggregates, we then sought to analyze our library of molecular simulation trajectories to resolve the molecular self-assembly pathways promoted by the high-performing peptide sequences to gain mechanistic understanding of the link between sequence and the emergent supramolecular structure. We hypothesized that the ensemble of simulation trajectories for 1181 different $X_{n}-4 \mathrm{~T}-X_{n}$ molecules collected over the course of our computational screen may admit a low-dimensional clustering within the configurational phase space of 
assembly pathways, and that the high-performing molecules should follow similar assembly pathways to reach the terminal pseudo-1D nanoaggregates. We report in Fig. 4a-b a 2D embedding into the leading two collective variables $\boldsymbol{\psi}_{2}$ and $\boldsymbol{\psi}_{3}$ discovered by diffusion maps. By correlating these collective variables with candidate physical observables, we find $\boldsymbol{\psi}_{2}$ to be strongly correlated with the instantaneous radius of gyration $R_{g}$ of the system $\left(\rho\left(\boldsymbol{\psi}_{2}, R_{g}\right)=0.93\right)$ and $\boldsymbol{\psi}_{3}$ moderately strongly correlated with the instantaneous number of contacts per molecule $\kappa\left(\rho\left(\boldsymbol{\psi}_{3}, \kappa\right)=0.69\right)$. In addition to providing good physical interpretability of the low-dimensional manifold containing the molecular assembly pathways learned by diffusion maps, the emergence of two collective variables strongly correlated with $\kappa$ and $R_{g}$ provides post hoc support for our selection of two observables as the dual objective functions of our computational screen as the leading variables governing the long-time self-assembly dynamics.

In Fig. 4c-f we illustrate the temporal evolution of the self-assembly pathways for particular $X_{n}-4 \mathrm{~T}-X_{n}$ molecules over the $\boldsymbol{\psi}_{2}-\boldsymbol{\psi}_{3}$ manifold. Each molecular trajectory begins at the right-most edge of the of the manifold corresponding to the initial monodisperse state. Lateral leftward movement across the manifold correspond to condensation of the system to smaller $R_{g}$ values due to the formation of nanoaggregates. Vertical upward movement corresponds to the accumulation of inter-molecular contacts between the $\pi$-cores and an elevation in $\kappa$. The assembly pathways of the top-performing candidates typically terminate in the upper-left corner of the manifold that contains pseudo-1D nanoaggreates containing $\kappa \approx 3$ intermolecular contacts and $R_{g} \approx 2 \mathrm{~nm}$ corresponding to elongated linear stacks. One prototypical class of assembly pathways for high-performing molecules is exemplified by EGG-4T-GGE (rank 4/28), which traverses the upper edge of the manifold (Fig. 4c). This assembly route corresponds to the rapid formation of small oligomeric stacks, the formation of which is likely promoted by the small size of the peptide wing, that ultimately associate into an elongated aggregate with good in-register and global stacking. DVAG-4T-GAVD (rank 11/28) is another high-performing candidate that is prototypical of a different assem- 

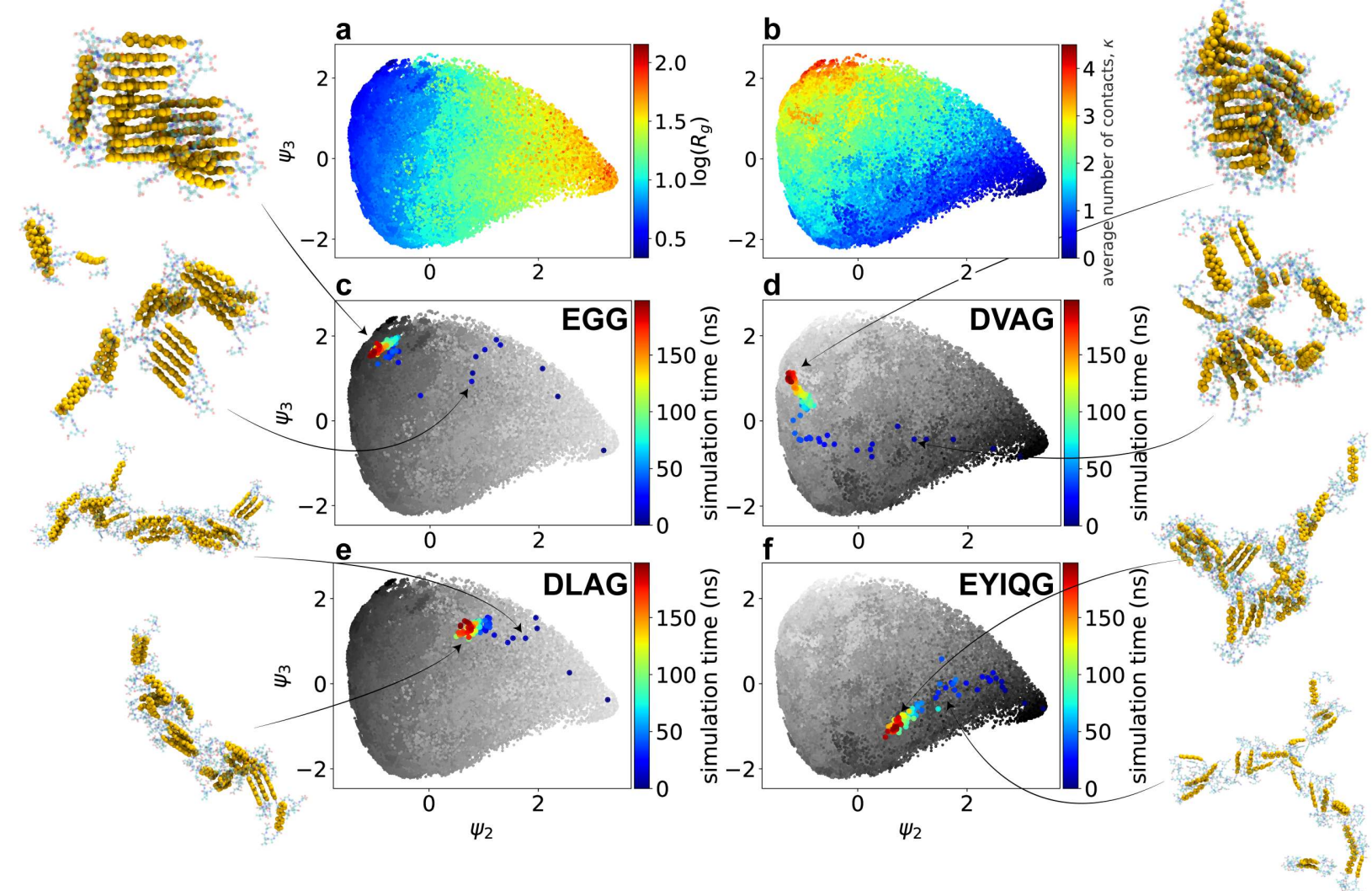

Figure 4: Two-dimensional diffusion map embedding of 118,100 simulation snapshots harvested from the molecular simulations of $1181 X_{n}-4 \mathrm{~T}-X_{n}$ molecules collected over the course of the computational active learning screen. Each point represents one simulation snapshot projected into a 2D low-dimensional manifold spanned by the leading collective variables $\boldsymbol{\psi}_{2}$ and $\boldsymbol{\psi}_{3}$ learned by diffusion maps. Coloring the points by (a) the log radius of gyration $\log \left(R_{g}\right)$ of the self-assembled nanoaggregate and (b) average number of contacts per molecule $\kappa$ within the aggregate exposes the strong correlation of the two learned collective variables with these two physical observables $\left(\rho\left(\boldsymbol{\psi}_{2}, R_{g}\right)=0.93, \rho\left(\boldsymbol{\psi}_{3}, \kappa\right)=0.69\right)$. All assembly trajectories commence in a monomeric dispersion contained at the rightmost edge of the manifold. Progression from right-to-left corresponds to a reduction in $R_{g}$ as the system selfassembles, and progression from bottom-to-top to the formation of more molecular contacts. The temporal progression of the self-assembly pathways over the manifold for molecules (c) EGG-4T-GGE, (d) DVAG-4T-GAVD, (e) DLAG-4T-GALD, and (f) EYIQG-4T-GQIYE, in which points are colored temporally. Grey points in panels c-f represent the embedding of 118,100 simulation snapshots shaded in panels $\mathrm{c}$ and e by $\log R_{g}$, and panels $\mathrm{d}$ and $\mathrm{f}$ by $\kappa$. Insets show representative molecular renderings throughout the trajectory.

bly route followed by high-performing molecules (Fig. 4d). This pathway commences with an initial rapid hydrophobic aggregation of the system corresponding to a rapid leftward lateral motion over the manifold. The absence of any early upward vertical motion is indicative of 
no initial substantive increase in $\kappa$ due to the larger peptide wings seemingly preventing good $\pi$-core stacking. This initial collapse is, however, then followed by a more gradual structural ripening as the cores do achieve good stacking and we observe late upward motion over the manifold corresponding to an increase in $\kappa$.

Trajectories that terminate within the bulk of the manifold and far away from the upperleft corner typically fail to form nanoaggregates containing globally connected pseudo-1D stacks. DLAG-4T-GALD (rank 24/28) is emblematic of a poor-performing molecule that initially builds a reasonable number of intermolecular contacts, but then fails to further condense into an in-register stacked nanoaggregate (Fig. 4e). Differing only in a V to L mutation relative to the high-performing DVAG-4T-GAVD, the presence of the bulkier hydrophobic Leu residue appears to preclude structural ripening into the desired elongated stack. Finally, molecules rich in large hydrophobic side chains such as EYIQG-4T-GQIYE (rank 18/28) tend to exhibit moderate leftward motion over the manifold corresponding to hydrophobic collapse but accompanied with unfavorable downward motion indicative of the formation of very few intermolecular $\pi$-core contacts (Fig. 4f). This behavior can be attributed to the bulky aromatic hydrophobes that stack against the $\pi$-cores and prevent the formation of core-core contacts.

Whereas Fig. 4 provided anecdotal insights into the self-assembly trajectories traced out by particular representative $X_{n}-4 \mathrm{~T}-X_{n}$ molecules, in Fig. 5 we present the entire distribution of trajectory end points for all molecules considered in our active learning screen. In Fig. 5ab we illustrate the end points of the 1181 molecules sampled in our computational screen colored by the $R_{g}$ and $\kappa$ values of the terminal nanoaggregates and in Fig. 5c the 28-molecule subset of these candidates that were experimentally tested colored by the measured spectral blue shift $\lambda$. Focusing on the 28 experimental molecules, we observe observe a clustering of 17 molecules in the upper left region of the manifold that we bound by a purple box. As anticipated by the understanding exposed by the diffusion map, these molecules tend to be high-performers comprising nine of the top 11 experimentally-tested molecules with spectral 
blue shifts $\lambda \geq 35 \mathrm{~nm}$. Further, the molecules within the box possess a mean spectral shift of $\lambda=31 \mathrm{~nm}$ compared to the mean value for those outside the box with $\lambda=21 \mathrm{~nm}$ (one-sided Mann-Whitney $\mathrm{U}$ test, $\mathrm{p}$-value $=0.07)$ The co-localization of $\left(\right.$ low- $R_{g}$-high $\left.\kappa\right)$ with high $\lambda$ within the diffusion map embedding provides a strong post hoc substantiation for the use of the former measures as a computational proxy for the latter within the active learning screen, and demonstrates the power and value of the high-throughput computational screen in focusing and guiding the low-throughput experimentation.

\section{Conclusions}

In this work, we have reported an integrated computational/experimental iterative design strategy to discover synthetic $\pi$-conjugated oligopeptides within the $X_{n}-4 \mathrm{~T}-X_{n}$ family with the capacity to self-assemble into highly-ordered linear aggregates with in-register stacking of the $\pi$-cores. These supramolecular assemblies are desirable as biocompatible nanoaggregates possessing emergent optoelectronic properties and potential applications as peptide-based field-effect transistors, photoconductors, or solar cells. The $X_{n}-4 \mathrm{~T}-X_{n}$ design space consisting of symmetric oligopeptide wings containing between one and five amino acids comprises 694,982 candidate molecules, making its exhaustive exploration impracticable by either simulation or experiment. By fusing computational and experimental data streams within an integrated computational-experimental active learning framework, we perform a data-driven efficient traversal the space of $X_{n}-4 \mathrm{~T}-X_{n}$ peptides that minimizes computational and experimental burden required to discover and validate new high-preforming candidates. Our platform employs a combination of all-atom molecular dynamics simulations, deep representational learning, single- and multi-fidelity Gaussian process regression, and single- and multi-objective Bayesian optimization. A computational active learning loop serves as a high-throughput and cheaply available experimental proxy used to refine a surrogate model that predicts the experimental performance of untested candidates. Using this platform, we 


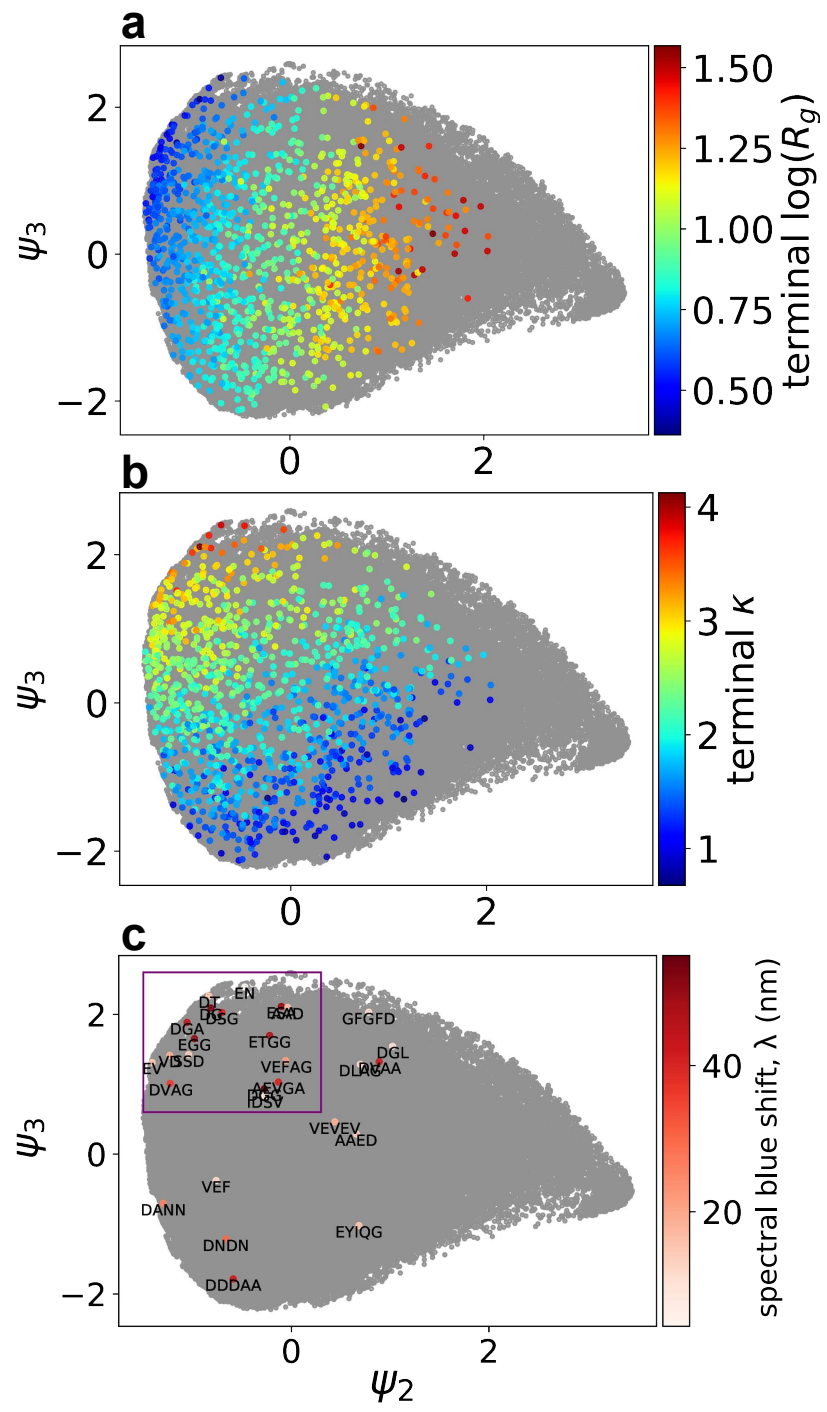

Figure 5: Terminal locations of the $X_{n}-4 \mathrm{~T}-X_{n}$ self-assembly trajectories over the 2D diffusion map manifold. End points of the 1181 molecules considered in our computational screen colored by the (a) $\log$ of the radius of gyration $\log \left(R_{g}\right)$ and (b) average number of contacts per molecule $\kappa$ computed over the terminal 50 ns of the trajectory. The 118,100 simulation snapshots used to construct the diffusion map embedding are shown in grey. (c) Terminal locations of the 28 experimentally tested molecules colored by their measured spectral blue shift $\lambda$ and annotated with the sequence of the peptide wing. The purple box bounds a cluster of high-performing experimental candidates residing in the upper-left region of the manifold possessing high values of $\lambda$.

discovered a diversity of high-performing new molecules experimentally validated to form pseudo-1D linear nanoaggregates after sampling only 1181 molecules $(\sim 0.17 \%$ of the design space) by computation and $28(\sim 0.004 \%)$ by experiment. Subsequent interrogation of our experimental screening data exposed molecular design rules linking sequence to the emergent 
structure and function of the self-assembled nanoaggregates. Analysis of the computational screening results revealed two prototypical assembly mechanisms and pathways shared by the high-performing molecules: (i) hierarchical assembly of small in-register supramolecular oligomers that undergo further assembly into a single linear aggregate with ordered $\pi$-stacking and (ii) rapid hydrophobic collapse followed by slow structural ripening and the emergence of in-register ordering of the $\pi$-cores.

This work provides a comprehensive simulation and experimental study of how variation in oligopeptide sequence in $\pi$-conjugated peptides impacts assembly behavior using an integrated experimental-computational active learning platform. Our findings corroborate prior physico-chemical understanding and chemical intuition of $\pi$-conjugated peptide assembly, but also reveals new design rules and understanding of molecular assembly mechanisms. Our hybrid computational/experimental active learning platform demonstrates the power of tightly integrated collaboration between theory and experiment, and this paradigm is transferable to other generic molecular design and discovery applications.

\section{Data Availability}

Data providing a full accounting of all molecules simulated and experimentally tested throughout the active learning process with associated measurements for the average number of contacts $\kappa$, radius of gyration $R_{g}$ and spectral blue shift $\lambda$, and terminal GPR and mfGPR predicted $\kappa, R_{g}$ and $\lambda$; neural network weights and training codes; GPR training codes; RAE embeddings; active learning workflow. ${ }^{33}$

\section{Conflict of Interest Disclosure}

A.L.F. is a co-founder and consultant of Evozyne, Inc. and a co-author of US Provisional Patents 62/853,919 and 62/900,420 and International Patent Applications PCT/US2020/035206 and PCT/US20/50466. 


\section{Acknowledgments}

This material is based upon work supported by the National Science Foundation under Grant Nos. DMR-1841807 and DMR-1728947, and a National Science Foundation Graduate Research Fellowship to K.S. under Grant No. DGE-1746045. This work was completed in part with resources provided by the University of Chicago Research Computing Center. We gratefully acknowledge computing time on the University of Chicago high-performance GPU-

based cyberinfrastructure supported by the National Science Foundation under Grant No. DMR-1828629. 


\section{References}

(1) Panda, S. S.; Katz, H. E.; Tovar, J. D. Solid-state electrical applications of protein and peptide based nanomaterials. Chemical Society Reviews 2018, 47, 3640-3658.

(2) Kumar, R. J.; MacDonald, J. M.; Singh, T. B.; Waddington, L. J.; Holmes, A. B. Hierarchical self-assembly of semiconductor functionalized peptide $\alpha$-helices and optoelectronic properties. Journal of the American Chemical Society 2011, 133, 8564-8573.

(3) Ardoña, H. A. M.; Tovar, J. D. Peptide $\pi$-electron conjugates: organic electronics for biology? Bioconjugate Chemistry 2015, 26, 2290-2302.

(4) Khalily, M. A.; Bakan, G.; Kucukoz, B.; Topal, A. E.; Karatay, A.; Yaglioglu, H. G.; Dana, A.; Guler, M. O. Fabrication of supramolecular n/p-nanowires via coassembly of oppositely charged peptide-chromophore systems in aqueous media. ACS Nano 2017, 11, 6881-6892.

(5) Stone, D. A.; Tayi, A. S.; Goldberger, J. E.; Palmer, L. C.; Stupp, S. I. Self-assembly and conductivity of hydrogen-bonded oligothiophene nanofiber networks. Chemical Communications 2011, 47, 5702-5704.

(6) Shao, H.; Seifert, J.; Romano, N. C.; Gao, M.; Helmus, J. J.; Jaroniec, C. P.; Modarelli, D. A.; Parquette, J. R. Amphiphilic self-assembly of an n-type nanotube. Angewandte Chemie 2010, 122, 7854-7857.

(7) Tsai, W.-W.; Tevis, I. D.; Tayi, A. S.; Cui, H.; Stupp, S. I. Semiconducting nanowires from hairpin-shaped self-assembling sexithiophenes. The Journal of Physical Chemistry $B$ 2010, 114, 14778-14786.

(8) Kitamura, T.; Nakaso, S.; Mizoshita, N.; Tochigi, Y.; Shimomura, T.; Moriyama, M.; Ito, K.; Kato, T. Electroactive supramolecular self-assembled fibers comprised of doped 
tetrathiafulvalene-based gelators. Journal of the American Chemical Society 2005, 127, $14769-14775$.

(9) Moghaddam, R. S.; Draper, E. R.; Wilson, C.; Heidari, H.; Adams, D. J. Effect of electric field on the electrical properties of a self-assembled perylene bisimide. $R S C$ Advances 2018, 8, 34121-34125.

(10) Eakins, G. L.; Pandey, R.; Wojciechowski, J. P.; Zheng, H. Y.; Webb, J. E.; Valéry, C.; Thordarson, P.; Plank, N. O.; Gerrard, J. A.; Hodgkiss, J. M. Functional organic semiconductors assembled via natural aggregating peptides. Advanced Functional Materials 2015, 25, 5640-5649.

(11) Pandeeswar, M.; Khare, H.; Ramakumar, S.; Govindaraju, T. Crystallographic insightguided nanoarchitectonics and conductivity modulation of an n-type organic semiconductor through peptide conjugation. Chemical Communications 2015, 51, 8315-8318.

(12) López-Andarias, J.; Rodriguez, M. J.; Atienza, C.; López, J. L.; Mikie, T.; Casado, S.; Seki, S.; Carrascosa, J. L.; Martín, N. Highly ordered n/p-co-assembled materials with remarkable charge mobilities. Journal of the American Chemical Society 2015, 137, 893-897.

(13) Lee, T.; Panda, S. S.; Tovar, J. D.; Katz, H. E. Unusually conductive organic-inorganic hybrid nanostructures derived from bio-inspired mineralization of peptide/pi-Electron assemblies. ACS Nano 2020, 14, 1846-1855.

(14) Besar, K.; Ardona, H. A. M.; Tovar, J. D.; Katz, H. E. Demonstration of hole transport and voltage equilibration in self-assembled $\pi$-conjugated peptide nanostructures using field-effect transistor architectures. ACS Nano 2015, 9, 12401-12409.

(15) Wall, B. D.; Zacca, A. E.; Sanders, A. M.; Wilson, W. L.; Ferguson, A. L.; Tovar, J. D. Supramolecular polymorphism: tunable electronic interactions within $\pi$-conjugated 
peptide nanostructures dictated by primary amino acid sequence. Langmuir 2014, 30, 5946-5956.

(16) Wall, B. D.; Diegelmann, S. R.; Zhang, S.; Dawidczyk, T. J.; Wilson, W. L.; Katz, H. E.; Mao, H.-Q.; Tovar, J. D. Aligned macroscopic domains of optoelectronic nanostructures prepared via shear-flow assembly of peptide hydrogels. Advanced Materials 2011, 23, 5009-5014.

(17) Panda, S. S.; Shmilovich, K.; Herringer, N. S.; Marin, N.; Ferguson, A. L.; Tovar, J. D. Computationally guided tuning of peptide-conjugated perylene diimide self-assembly. Langmuir 2021, DOI: 10.1021/acs.langmuir.1c01213.

(18) Panda, S. S.; Shmilovich, K.; Ferguson, A. L.; Tovar, J. D. Controlling supramolecular chirality in peptide- $\pi$-peptide networks by variation of the alkyl spacer length. Langmuir 2019, 35, 14060-14073.

(19) Horowitz, G. Organic field-effect transistors. Advanced Materials 1998, 10, 365-377.

(20) Mishra, A.; Ma, C.-Q.; Bauerle, P. Functional oligothiophenes: molecular design for multidimensional nanoarchitectures and their applications. Chemical Reviews 2009, 109, 1141-1276.

(21) Valverde, L. R.; Thurston, B. A.; Ferguson, A. L.; Wilson, W. L. Evidence for prenucleated fibrilogenesis of acid-mediated self-assembling oligopeptides via molecular simulation and fluorescence correlation spectroscopy. Langmuir 2018, 34, 7346-7354.

(22) Frederix, P. W.; Scott, G. G.; Abul-Haija, Y. M.; Kalafatovic, D.; Pappas, C. G.; Javid, N.; Hunt, N. T.; Ulijn, R. V.; Tuttle, T. Exploring the sequence space for (tri-) peptide self-assembly to design and discover new hydrogels. Nature Chemistry 2015, ๆ, 30. 
(23) Kim, C.; Chandrasekaran, A.; Jha, A.; Ramprasad, R. Active-learning and materials design: The example of high glass transition temperature polymers. MRS Communications 2019, 9, 860-866.

(24) Ling, J.; Hutchinson, M.; Antono, E.; Paradiso, S.; Meredig, B. High-dimensional materials and process optimization using data-driven experimental design with wellcalibrated uncertainty estimates. Integrating Materials and Manufacturing Innovation 2017, 6, 207-217.

(25) Barrett, R.; White, A. D. Investigating Active Learning and Meta-Learning for Iterative Peptide Design. Journal of Chemical Information and Modeling 2021, 61, 95-105.

(26) Gómez-Bombarelli, R.; Wei, J. N.; Duvenaud, D.; Hernández-Lobato, J. M.; SánchezLengeling, B.; Sheberla, D.; Aguilera-Iparraguirre, J.; Hirzel, T. D.; Adams, R. P.; Aspuru-Guzik, A. Automatic chemical design using a data-driven continuous representation of molecules. ACS Central Science 2018, 4, 268-276.

(27) Li, F.; Han, J.; Cao, T.; Lam, W.; Fan, B.; Tang, W.; Chen, S.; Fok, K. L.; Li, L. Design of self-assembly dipeptide hydrogels and machine learning via their chemical features. Proceedings of the National Academy of Sciences 2019, 116, 11259-11264.

(28) Nagasawa, S.; Al-Naamani, E.; Saeki, A. Computer-aided screening of conjugated polymers for organic solar cell: classification by random forest. The Journal of Physical Chemistry Letters 2018, 9, 2639-2646.

(29) Shmilovich, K.; Mansbach, R. A.; Sidky, H.; Dunne, O. E.; Panda, S. S.; Tovar, J. D.; Ferguson, A. L. Discovery of self-assembling $\pi$-conjugated peptides by active learningdirected coarse-grained molecular simulation. The Journal of Physical Chemistry B 2020, 124, 3873-3891.

(30) Thurston, B. A.; Ferguson, A. L. Machine learning and molecular design of selfassembling-conjugated oligopeptides. Molecular Simulation 2018, 44, 930-945. 
(31) Balachandran, P. V.; Kowalski, B.; Sehirlioglu, A.; Lookman, T. Experimental search for high-temperature ferroelectric perovskites guided by two-step machine learning. Nature Communications 2018, 9, 1-9.

(32) Gómez-Bombarelli, R.; Aguilera-Iparraguirre, J.; Hirzel, T. D.; Duvenaud, D.; Maclaurin, D.; Blood-Forsythe, M. A.; Chae, H. S.; Einzinger, M.; Ha, D.-G.; Wu, T. et al. Design of efficient molecular organic light-emitting diodes by a high-throughput virtual screening and experimental approach. Nature Materials 2016, 15, 1120-1127.

(33) Shmilovich, K.; Panda, S. S.; Stouffer, A.; Tovar, J. D.; Ferguson, A. L. Supporting data for: "Hybrid computational-experimental data-driven design of self-assembling $\pi$-conjugated peptides". 2021; https://doi.org/10.5281/zenodo. 5048397.

(34) Humphrey, W.; Dalke, A.; Schulten, K. VMD: visual molecular dynamics. Journal of Molecular Graphics 1996, 14, 33-38.

(35) Pareto, V. Cours d'économie politique; Librairie Droz, 1964; Vol. 1.

(36) Ghosh, P.; Sajjadi, M. S.; Vergari, A.; Black, M.; Schölkopf, B. From variational to deterministic autoencoders. arXiv preprint arXiv:1903.12436 2019,

(37) Rasmussen, C. E.; Williams, C. K. I. Gaussian processes for machine learning (adaptive computation and machine learning); The MIT Press, 2005.

(38) Brochu, E.; Cora, V. M.; De Freitas, N. A tutorial on Bayesian optimization of expensive cost functions, with application to active user modeling and hierarchical reinforcement learning. arXiv preprint arXiv:1012.2599 2010,

(39) Paria, B.; Kandasamy, K.; Póczos, B. A flexible framework for multi-objective Bayesian optimization using random scalarizations. Proceedings of The 35th Uncertainty in Artificial Intelligence Conference. 2020; pp 766-776. 
(40) Perdikaris, P.; Raissi, M.; Damianou, A.; Lawrence, N. D.; Karniadakis, G. E. Nonlinear information fusion algorithms for data-efficient multi-fidelity modelling. Proceedings of the Royal Society A: Mathematical, Physical and Engineering Sciences 2017, 473, 20160751.

(41) Abraham, M. J.; Murtola, T.; Schulz, R.; Páll, S.; Smith, J. C.; Hess, B.; Lindahl, E. GROMACS: High performance molecular simulations through multi-level parallelism from laptops to supercomputers. SoftwareX 2015, 1, 19-25.

(42) Mark, P.; Nilsson, L. Structure and dynamics of the TIP3P, SPC, and SPC/E water models at 298 K. The Journal of Physical Chemistry A 2001, 105, 9954-9960.

(43) Lindorff-Larsen, K.; Piana, S.; Palmo, K.; Maragakis, P.; Klepeis, J. L.; Dror, R. O.; Shaw, D. E. Improved side-chain torsion potentials for the Amber ff99SB protein force field. Proteins: Structure, Function, and Bioinformatics 2010, 78, 1950-1958.

(44) Bussi, G.; Donadio, D.; Parrinello, M. Canonical sampling through velocity rescaling. The Journal of Chemical Physics 2007, 126, 014101.

(45) Parrinello, M.; Rahman, A. Polymorphic transitions in single crystals: A new molecular dynamics method. Journal of Applied Physics 1981, 52, 7182-7190.

(46) Theodorou, D. N.; Suter, U. W. Shape of unperturbed linear polymers: polypropylene. Macromolecules 1985, 18, 1206-1214.

(47) Wang, J.; Ferguson, A. L. Mesoscale simulation of asphaltene aggregation. The Journal of Physical Chemistry B 2016, 120, 8016-8035.

(48) Mansbach, R. A.; Ferguson, A. L. Coarse-grained molecular simulation of the hierarchical self-assembly of $\pi$-conjugated optoelectronic peptides. Journal of Physical Chemistry B 2017, 121, 1684-1706. 
(49) Mansbach, R. A.; Ferguson, A. L. Control of the hierarchical assembly of $\pi$-conjugated optoelectronic peptides by $\mathrm{pH}$ and flow. Organic and Biomolecular Chemistry 2017, $15,5484-5502$.

(50) Mansbach, R. A.; Ferguson, A. L. Patchy particle model of the hierarchical self-assembly of $\pi$-conjugated optoelectronic peptides. The Journal of Physical Chemistry B 2018, 122, 10219-10236.

(51) Warmuth, M. K.; Liao, J.; Rätsch, G.; Mathieson, M.; Putta, S.; Lemmen, C. Active learning with support vector machines in the drug discovery process. Journal of Chemical Information and Computer Sciences 2003, 43, 667-673.

(52) Pertusi, D. A.; Moura, M. E.; Jeffryes, J. G.; Prabhu, S.; Biggs, B. W.; Tyo, K. E. Predicting novel substrates for enzymes with minimal experimental effort with active learning. Metabolic Engineering 2017, 44, 171-181.

(53) Varela, R.; Walters, W. P.; Goldman, B. B.; Jain, A. N. Iterative refinement of a binding pocket model: active computational steering of lead optimization. Journal of Medicinal Chemistry 2012, 55, 8926-8942.

(54) Khanna, V.; Ranganathan, S. In silico approach to screen compounds active against parasitic nematodes of major socio-economic importance. BMC bioinformatics. 2011; pp $1-12$.

(55) Reker, D.; Schneider, P.; Schneider, G. Multi-objective active machine learning rapidly improves structure-activity models and reveals new protein-protein interaction inhibitors. Chemical Science 2016, 7, 3919-3927.

(56) Naik, A. W.; Kangas, J. D.; Langmead, C. J.; Murphy, R. F. Efficient modeling and active learning discovery of biological responses. PLoS One 2013, 8, e83996. 
(57) Kingma, D. P.; Welling, M. Auto-encoding variational bayes. arXiv preprint $\operatorname{arXiv:1312.61142013,}$

(58) Gilmer, J.; Schoenholz, S. S.; Riley, P. F.; Vinyals, O.; Dahl, G. E. Neural message passing for quantum chemistry. International Conference on Machine Learning. 2017; pp 1263-1272.

(59) Battaglia, P. W.; Hamrick, J. B.; Bapst, V.; Sanchez-Gonzalez, A.; Zambaldi, V.; Malinowski, M.; Tacchetti, A.; Raposo, D.; Santoro, A.; Faulkner, R. et al. Relational inductive biases, deep learning, and graph networks. arXiv preprint arXiv:1806.01261 2018

(60) Chung, J.; Gulcehre, C.; Cho, K.; Bengio, Y. Empirical evaluation of gated recurrent neural networks on sequence modeling. arXiv preprint arXiv:1412.3555 2014,

(61) Li, Y.; Tarlow, D.; Brockschmidt, M.; Zemel, R. Gated graph sequence neural networks. arXiv preprint arXiv:1511.05493 2015,

(62) Vinyals, O.; Bengio, S.; Kudlur, M. Order matters: Sequence to sequence for sets. arXiv preprint arXiv:1511.06391 2015,

(63) Kawashima, S.; Pokarowski, P.; Pokarowska, M.; Kolinski, A.; Katayama, T.; Kanehisa, M. AAindex: amino acid index database, progress report 2008. Nucleic Acids Research 2007, 36, D202-D205.

(64) Kingma, D. P.; Ba, J. Adam: A method for stochastic optimization. arXiv preprint arXiv:1412.6980 2014,

(65) Paszke, A.; Gross, S.; Massa, F.; Lerer, A.; Bradbury, J.; Chanan, G.; Killeen, T.; Lin, Z.; Gimelshein, N.; Antiga, L. et al. Pytorch: An imperative style, highperformance deep learning library. Advances in Neural Information Processing Systems 2019, 32, 8026-8037. 
(66) Auer, P. Using confidence bounds for exploitation-exploration trade-offs. Journal of Machine Learning Research 2002, 3, 397-422.

(67) Kasha, M.; Rawls, H.; El-Bayoumi, M. A. The exciton model in molecular spectroscopy. Pure and Applied Chemistry 1965, 11, 371-392.

(68) Ardoña, H. A. M.; Besar, K.; Togninalli, M.; Katz, H. E.; Tovar, J. D. Sequencedependent mechanical, photophysical and electrical properties of pi-conjugated peptide hydrogelators. Journal of Materials Chemistry C 2015, 3, 6505-6514.

(69) Lizotte, D. J. Practical Bayesian optimization. Ph.D. thesis, 2008.

(70) Močkus, J. On Bayesian methods for seeking the extremum. Optimization techniques IFIP technical conference. 1975; pp 400-404.

(71) Ginsbourger, D.; Le Riche, R.; Carraro, L. A multi-points criterion for deterministic parallel global optimization based on Gaussian processes. HAL preprint hal-00260579 2008 ,

(72) Coifman, R. R.; Lafon, S. Diffusion maps. Applied and Computational Harmonic Analysis 2006, 21, 5-30.

(73) Nadler, B.; Lafon, S.; Coifman, R. R.; Kevrekidis, I. G. Diffusion maps, spectral clustering and eigenfunctions of Fokker-Planck operators. arXiv preprint math/0506090 2005

(74) Long, A. W.; Ferguson, A. L. Rational design of patchy colloids via landscape engineering. Molecular Systems Design \& Engineering 2018, 3, 49-65.

(75) Wang, J.; Ferguson, A. L. Nonlinear machine learning in simulations of soft and biological materials. Molecular Simulation 2018, 44, 1090-1107.

(76) Ma, Y.; Ferguson, A. L. Inverse design of self-assembling colloidal crystals with omnidirectional photonic bandgaps. Soft Matter 2019, 15, 8808-8826. 
(77) Ferguson, A. L.; Panagiotopoulos, A. Z.; Debenedetti, P. G.; Kevrekidis, I. G. Systematic determination of order parameters for chain dynamics using diffusion maps. Proceedings of the National Academy of Sciences 2010, 107, 13597-13602.

(78) Bartók, A. P.; Kondor, R.; Csányi, G. On representing chemical environments. Physical Review B 2013, 87, 184115.

(79) Himanen, L.; Jäger, M. O.; Morooka, E. V.; Canova, F. F.; Ranawat, Y. S.; Gao, D. Z.; Rinke, P.; Foster, A. S. DScribe: Library of descriptors for machine learning in materials science. Computer Physics Communications 2020, 247, 106949.

(80) De, S.; Bartók, A. P.; Csányi, G.; Ceriotti, M. Comparing molecules and solids across structural and alchemical space. Physical Chemistry Chemical Physics 2016, 18, 1375413769.

(81) Jäger, M. O.; Morooka, E. V.; Canova, F. F.; Himanen, L.; Foster, A. S. Machine learning hydrogen adsorption on nanoclusters through structural descriptors. npj Computational Materials 2018, 4, 1-8.

(82) Wang, J.; Gayatri, M. A.; Ferguson, A. L. Mesoscale simulation and machine learning of asphaltene aggregation phase behavior and molecular assembly landscapes. The Journal of Physical Chemistry B 2017, 121, 4923-4944.

(83) Thurston, B. A.; Shapera, E. P.; Tovar, J. D.; Schleife, A.; Ferguson, A. L. Revealing the sequence-structure-electronic property relation of self-assembling $\pi$-conjugated oligopeptides by molecular and quantum mechanical modeling. Langmuir 2019, 35, $15221-15231$.

(84) Mann, H. B.; Whitney, D. R. On a test of whether one of two random variables is stochastically larger than the other. The Annals of Mathematical Statistics 1947, 5060. 
(85) Hartgerink, J. D.; Beniash, E.; Stupp, S. I. Self-assembly and mineralization of peptideamphiphile nanofibers. Science 2001, 294, 1684-1688.

(86) Sanders, A. M.; Dawidczyk, T. J.; Katz, H. E.; Tovar, J. D. Peptide-based supramolecular semiconductor nanomaterials via Pd-catalyzed solid-phase "dimerizations". ACS Macro Letters 2012, 1, 1326-1329.

(87) Vadehra, G. S.; Wall, B. D.; Diegelmann, S. R.; Tovar, J. D. On-resin dimerization incorporates a diverse array of $\pi$-conjugated functionality within aqueous self-assembling peptide backbones. Chemical Communications 2010, 46, 3947-3949.

(88) Panda, S. S.; Tovar, J. D. Aqueous self-assembly of peptide-diketopyrrolopyrrole conjugates with variation of N-alkyl side chain and $\pi$-core lengths. Organic Materials 2021,

(89) Panda, S. S.; Shmilovich, K.; Ferguson, A. L.; Tovar, J. D. Computationally guided tuning of amino acid configuration influences the chiroptical properties of supramolecular peptide- $\pi$-peptide nanostructures. Langmuir 2020, 36, 6782-6792. 


\section{TOC Graphic}

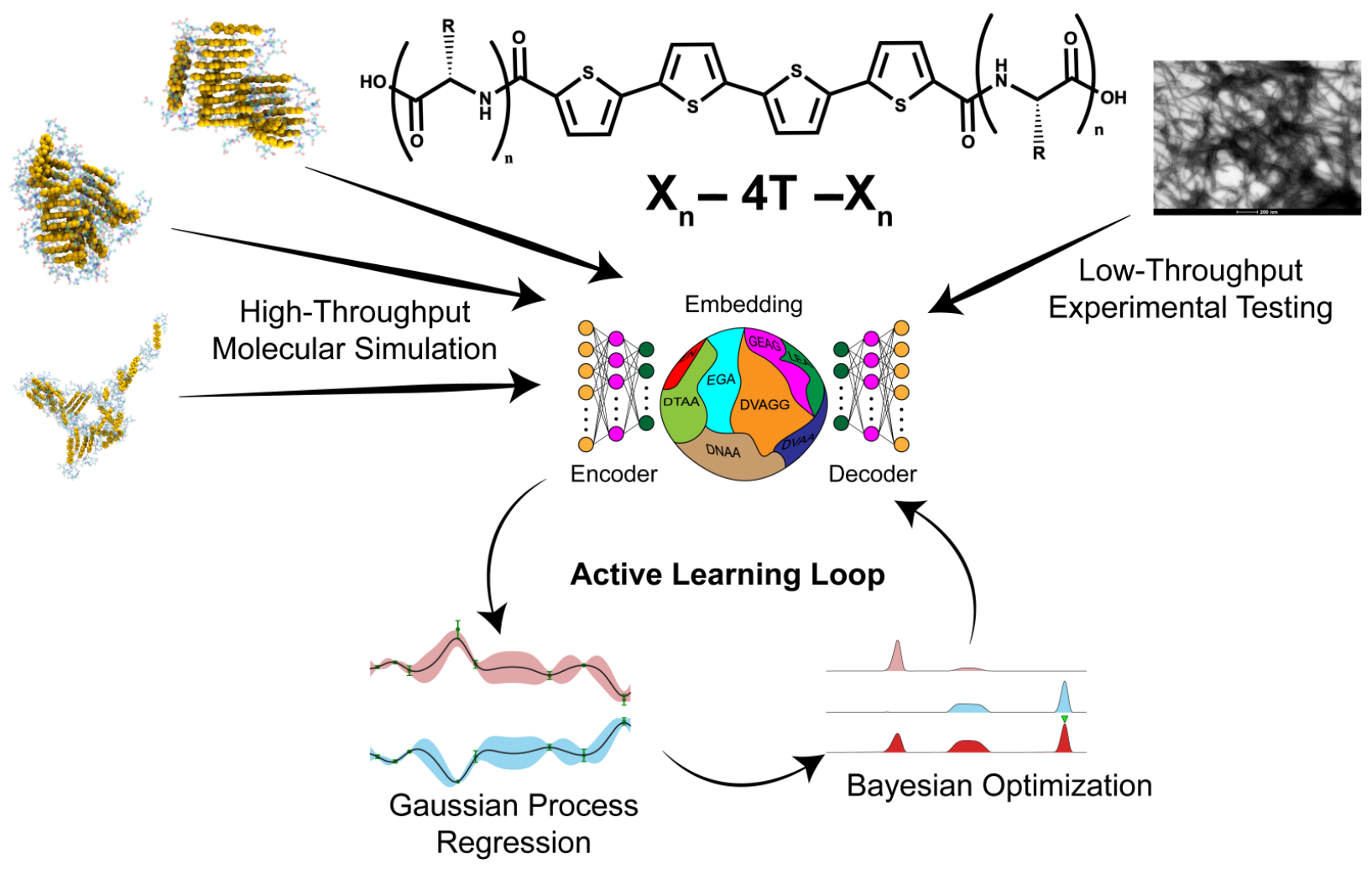

\section{Synopsis}

A hybrid computational-experimental active learning platform enables efficient discovery of bio-compatible $\pi$-conjugated peptides programmed to self-assemble into conductive elongated nanofibers. 